\title{
GENERALIZED GROUP ALGEBRAS AND THEIR BUNDLES
}

\author{
I.E. SCHOCHETMAN \\ Department of Mathematical Sciences, Oakland University \\ Rochester, Michigan 48063 \\ (Recelved September 16, 1980, and in revised form July 20, 1981)
}

ABSTRACT. Our primary objective here is to extend the concept of Banach *-algebraic bundle to the setting where the bundle product and involution are just measurable, i.e. not necessarily continuous. Our secondary objective is to introduce the *-algebra operations into such a bundle by means of operator fields and study the smoothness of these operations in terms of the smoothness of the fields.

KEY WORDS AND PHRASES. Fields of Banach spaces, continuity structures, Banach bundles, induced continuity structures, bundle morphisms, product fields, involution fields, group-algebra bundles, bundle identities, bundle multipliers, generalized group-algebras, homogeneous bundles, unitary factor systems, twisting pairs, group-extension bundles.

1980 MATHEMATICS SUBJECT CLASSIFICATION CODES. Primary: 46H99, 46K99. Secondary: 22015, 43A20, 55FTO.

\section{INTRODUCTION.}

Let $G$ be a locally compact group and $A$ a Banach $*$-algebra. The question we are interested in here is how to construct a Banach *-algebra from $L^{1}(G, A)$ which generalizes the canonical one (with pointwise operations). Alternately, how generally can we introduce multiplication and involution in $L^{1}(G, A)$ so as to make it a Banach *-algebra? The most general existing responses to this question are quite different. They are:

(1) The cross-section algebras of Fell [1,2] and their underlving Banach *-algebraic bundles.

(2) The generalized $L^{1}$-algebras of Leptin [3] or the equivalent twisted 
group algebras of Busby and Smlth [4]. The underlying Banach bundles in these constructions are trivial (see [5]).

Nefther of the previous approaches 18 more general than the other. However, they were designed for the same purpose. Thus, 1t seems desirable that they be unified as part of a more general theory which includes them both--as well as their bundles. This is our overall goal in this manuscript.

Since multiplication and involution are fust measurable in the trivial bundles associated with the Leptin and Busby-Smith constructions, this should be true of the bundles in the general theory. This is the main shortcoming of the Fell approach via bundles. On the other hand, since Fell's approach allows for non-1somorphic flbers In the bundles (1.e. non-trivial bundles), this should also be true of the bundles in the general theory. This is the main shortcoming of the Leptin and Busby-Smith constructions. Consequently, our primary objective is to extend Fell's Banach *-algebralc bundle approach to the setting where the multiplication and Involution need only be measurable. This extension will then include the Leptin and Busby-Smith constructions. However, the method by which we realize the measurable *-algebra structure in a (Banach) bundle w11l be quite different from that of Fell. In fact, 1t will be more consistent with the other two approaches in that it will be done by means of operator flelds. This is our secondary objective. There 1s good reason for our choosing this approach which is explained very well by some recent remarks of Rieffel [6]:

"Fell's approach in terms of bundles has some great advantages over those used in varlous other papers concerned with establishing a general framework,... in that Fell can work everywhere with continuous functions, thus avolding many measuretheoretic arguments, and he has no need to become entangled in lengthy cocycle computations and the like. On the other hand, in many speciflc situations which one may want to study, the bundle structure is often not entirely evident so that the translation between the immediately evident structure and Fell's bundle structure may be tedious. Thus while the theory developed by Fell in these notes is of very considerable philosophical comfort, more experience will be needed before it will be clear 
exactly how incisive a technical tool it is for dealing with specific examples." To elaborate on Rieffel's remarks, observe that in the Fell construction the bundle structure is introduced axiomatically. On the other hand, in the Leptin and Busby-Smith constructions, the bundle structure is introduced operator-theoretically. The latter approach is characteristic of how the "immediately evident" bundle structure arises in specific examples. Thus, in short, our aim is to develop a general theory of group algebras via bundles which includes the existing theories and utilizes their respective advantages.

Taking all the above facts and comments into consideration, we have chosen to proceed as follows. Replace $A$ by a field $\left\{A_{x}: x \in G\right\}$ of Banach spaces indexed by G - In Section 1, we review the notion of continuity structure $\Lambda$ in $\operatorname{IA}_{x}$ and the equivalent notion of Banach bundle. In Section 2, we study certain continuity structures which can be derived from $\Lambda$--the induced structures. The notion and properties of bundle morphism are developed in Section 3. Then the notions of product and involution are introduced in Sections 4 and 5 by means of operator fields $P$ and I having the appropriate algebraic properties. By requiring these fields to be continuous (resp. measurable, ultra-measurable), we obtain a continuous (resp. measurable, ultra-measurable) Banach *-algebraic bundle. A group-algebra bundle is defined to be a measurable Banach *-algebraic bundle and is studied in Section 6. Section 7 studies to what extent the various notions and properties of bundle approximate identity extend from the continuous to the measurable case. Section 8 does the same for multipliers. In Section 9, we construct the $\mathrm{L}^{1}$-algebra corresponding to $G,\left\{A_{x}\right\}, \Lambda, P, I \cdot$ In Section 10, we investigate the extent to which bundle homogeneity extends from the continuous to the measurable case. Unitary factor systems and twisting pairs, as well as their bundles and corresponding algebras, are reviewed and compared in Sections 11 and 12, Next we construct the bundle corresponding to a locally compact group extension $1 \rightarrow N \rightarrow H \rightarrow G \rightarrow 1$. Fell's original construction was direct, in that he specified the bundle ingredients and then verified the equivalence of $\mathrm{L}^{1}(\mathrm{H})$ with the resulting cross-section algebra. In Section 13, we show that these ingredients arise naturally as a consequence of 
our $L^{1}$-induction procedure for regular representations. The equivalence of $L^{1}(H)$ with the cross-section algebra is then an automatic consequence of induction-instages.

In what follows, it will be convenient to use the following general notation. The symbols $\mathbf{X}, \mathbf{z}, \mathbb{R}, \mathbb{C}$ will denote (as usual) the natural numbers, integers, real numbers, complex numbers respectively. If $S$ is any set, then $E_{S}$ will denote the identity mapping on $S$ and $\gamma_{S}$ its characteristic function. If $A$ and $B$ are Banach spaces, then $\operatorname{Hom}(A, B)$ will denote the Banach space of bounded 1inear operators from $A$ into $B$. If $X$ is a locally compact Hausdorff space and $\mu$ is a positive Radon measure on $X$, then $C(X, A)$ (resp. $C_{C}(X, A)$ ) will denote the linear space of continuous (resp. compactly supported) A-valued functions on $\mathrm{X}$ and $M(X, A, \mu)$ the linear space of (equivalence classes of) such $\mu$-measurable functions. The phrase "for $\mu$-almost all $x$ in $G$ " will be abbreviated by " $\mu-a . a . x$ in G."

\section{CONTINUTTY STRUCTURES.}

Let $X$ be a locally compact Hausdorff space and $\left\{A_{x}: x \in X\right\}$ a field of Banach spaces over $x$. Let $A$ denote the disjoint union of the $A_{x}, x \in X:$ Define $\pi:, 1 \rightarrow x$ by $\pi^{-1}(x)=A_{x}, x \in X$. Note that $\Pi_{x}$ is a linear space. An element of $\Pi A_{x}$ will be called a vector field. Of fundamental significance here is the notion of continuity for a vector field. Since there is no canonical meaning for this notion in general, it must be introduced axiomatically. This was first accomplished by Godement in [7] (see also [8]) by means of a "continuity structure." Actually, Godement's original terminology was "fundamental family of continuous vector fields." The term "continuity structure" is taken from Fell [9].

DEFINITION 1.1. A continuity structure $\Lambda$ in $\Pi_{x}$ is a subset satisfying:

(i) $\Lambda$ is a subspace of $\pi_{x}$.

(ii) For each $h$ in $\Lambda$, the scalar function $x \rightarrow h(x) \|$ is continuous on $x$. (iii) For each $x$ in $X$, the subspace $\{h(x): h \varepsilon \Lambda\}$ of $A_{X}$ is dense.

Given a continuity structure, we define continuity for a vector field as follows : 
DEFINITION 1.2. Let $x_{0} \varepsilon D S X$ and $f \varepsilon \Pi_{D} A_{x}$. Then $f$ is $\Lambda$-continuous at $x_{0}$ if, given $\varepsilon>0$, there exists a neighborhood $N$ of $x_{0}$ in $X$ and $h$ in $\Lambda$ such that $\|f(x)-h(x)\|<\varepsilon$, for $x$ in $N M D$. The field $f$ (defined on $D$ ) is $\Lambda$-continuous if it is so at each point of $D$. Denote the space of such fields by $C(D, \Lambda)$; if $D=X$, we write $C(\Lambda)$ for $C(X, \Lambda)$. Clearly, $\Lambda \subseteq C(\Lambda)$ in general. Hence, in this context, to say that $f$ is a continuous vector field means that $f$ is an element of the linear space $C(\Lambda)$. (See sections 1 and 5 of [8].) Let $C_{c}(\Lambda)=\{f \varepsilon C(\Lambda): f$ has compact support $\}$.

DEFINITION 1.3. A subset $\Gamma$ of $\Lambda$ is total if $\{h(x): h \varepsilon \Gamma\}$ is dense in $A_{x}$, for each $x$ in $x$. Thus, $\Lambda$ itself is total.

LEMMA 1.4. Let $\Gamma$ be total in $\Lambda$. In $1.2, f$ is $\Lambda$-continuous at $x_{0}$ if and only if the element $h$ in $\Lambda$ can be chosen from $\Gamma$.

Some time after Godement, Fell introduced the notion of continuity into our context in a very different way--by axiomatically topologizing A[1]. For this purpose, it will be convenient to speak of the elements of $\Pi_{x}$ as cross-sections and denote the space of them by $S(X, A)$.

DEFINITION 1.5. (A, $\pi)$ is a Banach bundle over $X$ if $A$ is a Hausdorff space, $\pi$ is a continuous, open surjection and:

(i) The function $a \rightarrow\|a\|$ is continuous on $A$.

(ii) The operation + is continuous from

$$
\{(a, b) \varepsilon A \times A: \pi(a)=\pi(b)\}
$$

into $A$.

(iii) For each $B$ in $\boldsymbol{C}$, the mapping on $A$ given by $a \rightarrow B a$ is continuous.

(iv) If $x \in X$ and $\left\{a_{i}\right\}$ is a net in $A$ such that $\left\|a_{i}\right\| \rightarrow 0$ and $\pi\left(a_{i}\right) \rightarrow x$ in $X$ then $a_{i} \rightarrow 0_{x}$ (the zero of $A_{x}$ ) in $A$.

Let $\operatorname{CS}(X, A)$ denote the subset of $S(X, A)$ consisting of continuous cross-sections. Observe that the relative topology of $A$ on each fiber $A_{x}$ is precisely the norm topology [1, p.10]. More importantly, we have the following:

LEMMA 1.6. The space $\operatorname{CS}(\mathrm{X}, \mathrm{A})$ is a continuity structure in $\mathrm{IIA}_{\mathrm{X}}$ with 
equality holding in part (iil) of 1.1 .

PROOF. Part (i) of 1.1 follows from [1, p. 11]. Part (1i) is clear. Part (iii) is a very recent development and follows from the fact that $(A, \pi)$ has enough continuous cross-sections [1, Thm. 11].

Letting $\Lambda=\operatorname{cs}(X, A)$, we obtain that $C(\Lambda)=\Lambda[8, p .13]$. Therefore, startIng with a Banach bundle, we obtain a continuity structure in $\pi A_{x}$ which is the largest such structure in 1ts equivalence class [8, Prop. 1.23]. (Recall that $\Lambda_{1} \sim \Lambda_{2}$ if $\mathrm{C}\left(\Lambda_{1}\right)=\mathrm{C}\left(\Lambda_{2}\right)$.) Conversely, it has been known for some time that this process is reversible (up to equivalence).

LEMMA 1.7. Let $\Lambda$ be a continuity structure in $\pi_{x}$. Then there exists a unique topology on $A$ making $(A, \pi)$ a Banach bundle. Furthermore,

$$
\Lambda \subseteq \operatorname{cs}(x, A) \text {; in fact, } C(\Lambda)=\operatorname{cs}(x, A)
$$

LEMMA 1.8. Let $x_{0} \varepsilon D \subseteq X$ and $f \in \Pi_{D} A_{x}$, so that $f: D \rightarrow A$. Then the crosssection $f$ is $A$-continuous at $x_{0}$ if and only if the vector field $f$ is $\Lambda$-continuous at $x_{0}$. Hence, $C(D, \Lambda)=\operatorname{cs}(D, A)$.

Thus, starting with a continuity structure $\Lambda$, we obtain a Banach bundle $(A, \pi)$ for which $\Lambda \sim \operatorname{CS}(X, A)$. This shows that the two methods for obtaining continuous vector fields in $\pi_{x}$ are equivalent, 1.e. $C(\Lambda)=\operatorname{cs}(x, A)$. For the remainder of this paper, we will let $\Lambda$ be a continuity structure in $\pi A_{x}$ and $(A, \pi)$ the unique Banach bundle guaranteed by 1.7. In particular, if $X$ is discrete then $\Pi_{x}$ itself is the essentially unique continuity structure $[8,1.22]$ in $\Pi_{x}$. Next we turn to the notion of measurability. Let $\mu$ be a positive Radon measure on $X$. First we consider the vector field context.

DEFINITION 1.9. Let $\mathrm{D}$ be a locally compact subspace of $\mathrm{X}$ and $\mathrm{f}$ an element of $\Pi_{D} A_{x}$. Then $f$ is $(\Lambda, \mu)$-measurable if, given compact $K \subseteq x$ and $\varepsilon>0$, there exists compact $K_{\varepsilon} \subseteq K$ such that $\mu\left(K-K_{\varepsilon}\right)<\varepsilon$ and $f$ is $\Lambda$-continuous on $K_{\varepsilon}$. Denote such $f$ by $M(D, \Lambda, \mu)$. If $D=X$, delete $i t$.

Analogously, we wish to introduce measurability in $S(X, A)$ (as in [10]). 
DEFINITION 1.10. Let $D$ and $f$ be as in 1.9 . Then $f$ is $(A, \mu)-$ messurable 1f, given compact $K \subseteq D$ and $\varepsilon>0$, there exists compact $K_{\varepsilon} \mathcal{K}$ such that $\mu\left(K-K_{\varepsilon}\right)<\varepsilon$ and $f: K_{\varepsilon}+A$ 1s continuous for the $\Lambda$-topology of $A$. Denote such $f$ by $\operatorname{MS}(D, A, \mu)$.

REMARK 1.11. On p. 22 of [1], Fell defines the notion of measurable crosssection in a different way. However, as can be verified by the results on p. 23 of [1], the two definitions are equivalent.

LEMMA 1.12. If $D$ 1s a locally compact subset of $X$, then

$$
M S(D, A, \mu)=M(D, \Lambda, \mu) \text {. }
$$

In particular,

$$
\operatorname{MS}(X, A, \mu)=M(\Lambda, \mu)
$$

PROOF. Th1s follows from 1.8 .

REMARK 1.13. Since continulty and $\mu$-measurab1lity depend only on the equivalence class of $\Lambda[8,551,3]$, we may replace $\Lambda$ by $C(\Lambda)=\operatorname{CS}(X, A)$ without 1088 of generality. (Of course, this 18 false if we find it necessary to consider equicontinuous familles of vector flelds [8, \$2]). Thus, we may (and w111) assume $\Lambda=C(\Lambda)=\operatorname{CS}(X, A)$. Actually, this 18 advantageous because of the last equality, as well as the fact that $\{h(x): h \varepsilon C(\Lambda)\}$ is equal to $A_{x}$, for $x$ in $x$. In what follows, $c(\Lambda)$ or $\Lambda$ will be used according to wh1ch 18 approprlate when they are not assumed to be equal.

The remainder of this section 18 devoted to separability considerations.

DEFINITION 1.14. [1, P.15]. The Banach bundle $(A, \pi)$ 1s strongly separable 1f $X$ is second countable and $\Lambda$ is countably dense, 1.e. $\Lambda$ contains a countable, total subset $[8,1.1]$.

LEMMA 1.15. The bundle $(A, \pi)$ is strongly separable if and only if it is second countable.

PROOF. This 1s Prop. 1.8 of [1]. Recall that $(A, \pi)$ automatically has enough cross-sections. 
Suppose for the moment that $\Lambda$ is arbitrary again, i.e. $\Lambda \subseteq c(\Lambda)$ in general. Recall [8, p.10] also that $\Lambda$ is separable if $C(\Lambda)$ is countably dense and $\Lambda$ is locally separable if, for each compact $K \Phi X$, the restricted continuity structure $\Lambda \mid K[8, p .9]$ is separable. In this context, we have: A strongly separable implies $\Lambda$ separable. The converse is false in general. However, if we again assume $\Lambda=C(\Lambda)$, then: $\Lambda$ is separable if and only if $\Lambda$ is countably dense. Therefore, under this assumption, we have the following separability summary:

LEMMA 1.16. The following are equivalent:

(i) $A$ is second countable, i.e. strongly separable.

(ii) $\Lambda$ is countable dense and $X$ is second countable.

(iii) $\Lambda$ is separable and $X$ is second countable.

EXAMPLE 1.17. Let

$$
1 \rightarrow \mathrm{N} \rightarrow \mathrm{H} \rightarrow \mathrm{G} \rightarrow 1
$$

be an exact sequence of locally compact groups. If $H$ is second countable, then so is $G$ and the group algebra $\mathrm{L}^{1}(\mathrm{~N})$ is separable. In Section 9, we will construct a Banach bundle over $G$ with fibers isometrically isomorphic to $L^{1}(N)$. In this case, the continuity structure will be countably dense, so that the Banach bundle will be second countable.

\section{INDUCED CONTINUITY STRUCTURES.}

The objectives of subsequent sections suggest that we study certain continuity structures "induced" from given ones. This section is devoted to defining these structures and establishing their basic properties for use later on.

Suppose $\left\{B_{\mathrm{y}}: \mathrm{y} \varepsilon \mathrm{Y}\right\}$ is a field of Banach spaces over the locally compact Hausdorff space $\mathrm{Y}$ with $\Delta$ a continuity structure in $\Pi_{\mathrm{y}}$. Let $(B, \tau)$ denote the corresponding bundle. Suppose also that $\psi: X \rightarrow Y$ is a continuous mapping from $X$ into $Y$. The induced bundle $[11]\left(B^{\psi}, \tau^{\psi}\right)$ over $X$ is then given by:

$$
\begin{aligned}
& B^{\psi}=\{(\mathrm{x}, \mathrm{b}): \mathrm{x} \varepsilon \mathrm{x}, \mathrm{b} \varepsilon B, \tau(\mathrm{b})=\psi(\mathrm{x})\}, \\
& \tau^{\psi}(\mathrm{x}, \mathrm{b})=\mathrm{x}, \quad(\mathrm{x}, \mathrm{b}) \varepsilon B^{\psi}
\end{aligned}
$$


We then have the following commutative diagram:

$$
\begin{array}{r}
B^{\psi} \rightarrow B \\
\tau^{\psi} \begin{array}{l}
\downarrow \\
\mathrm{X}_{\psi} \rightarrow \mathrm{Y}
\end{array}
\end{array}
$$

where the top mapping is the projection $(x, b) \rightarrow b$. (Also see [2, p.101] in this regard.) The set $B^{\psi}$ is (roughly) the disjoint union of the field $\left\{B_{\psi(x)}: x \in X\right\}$. If $\psi$ is one-to-one, then $B^{\psi}$ can be identified with $\tau^{-1}(\psi(X))$.

The cross-sections $S\left(X, B^{\psi}\right)$ are in one-to-one correspondence with

$$
\Pi_{\psi(x)}=\left\{f: X \rightarrow B: f(x) \varepsilon B_{\psi(x)}, x \in X\right\}
$$

via the mapping $f \leftrightarrow\left(E_{X}, f\right)$. For such $f$, the diagram

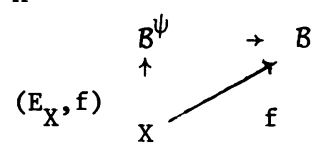

is commutative.

Suppose $B^{\psi}$ is equipped with the relativized topology of $\mathrm{X} \times B$.

LEMMA 2.1. Let $\Delta^{\psi}=\left\{\mathrm{E}_{\mathrm{X}} \times \mathrm{k} \psi: \mathrm{k} \varepsilon \Delta\right\}$. Then $\Delta^{\psi}$ is a continuity structure in $\Pi_{\psi(\mathbf{x})} \cdot$

LEMMA 2.2. Let $f \in \Pi_{D} B_{\psi(x)}$ for $D \subseteq X$ and $x_{0} \in D$. Then the following are equivalent:

(i) The vector field $\left(E_{X}, f\right)$ is $\Delta^{\psi}$-continuous at $x_{0}$.

(ii) The cross-section $\left(E_{X}, f\right)$ in $S\left(D, B^{\psi}\right)$ is continuous at $x_{0}$.

From 2.2, it follows that

$$
\begin{aligned}
\operatorname{cs}\left(X, B^{\psi}\right) & =C\left(\Delta^{\psi}\right) \\
& =\left\{\left(E_{X}, f\right): f \varepsilon C(X, B), f(x) \varepsilon B_{\psi(x)}, x \varepsilon X\right\}
\end{aligned}
$$

and

$$
\operatorname{MS}\left(\mathrm{X}, B^{\psi}, \mu\right)=M\left(\Delta^{\psi}, \mu\right)
$$

EXAMPLE 2.3. If $X \subseteq Y$ and $\psi$ is the injection mapping, then $\Delta^{\psi}$ is simply the restriction $\Delta \mid \mathrm{X}$ of $\Delta$ to $\mathrm{X}[8, \mathrm{p} .9]$. In this case, $B^{\psi}$ is homeomorphic to to $\tau^{-1}(X) \subseteq B$ and $\tau^{\psi}=\tau \mid B^{\psi}$. 
EXAMPLE 2.4. If $\psi: X+Y$ is a homemorphism, then $B^{\psi}=B$ and $\tau^{\psi}=\psi^{-1} \tau$ essentially. of course, $c\left(\Delta^{\psi}\right) \leftrightarrow c(\Delta)$, 1.e. $\left(E_{X}, h \psi\right) \leftrightarrow h$, hec $(\Delta)$.

EXAMPLE 2.5. If $\psi: Y \times X+Y$ is the left projection $\lambda$, then $\Delta^{\lambda}$ is the lifted continuity structure obtained from $\Delta[8, \S 4]$. The corresponding bundle $\left(B^{\lambda}, \tau^{\lambda}\right)$ can be Identified with $\left(X \times B, E_{X} \times \tau\right) \cdot$ (See p.27 of [1].)

3. BUNDLE MORPHISMS .

In later sections, it w11l be necessary to identify bundles (up to 1somorphism) as well as consider products of bundles in studying multiplication. Accordingly, we need to develop suitable notions about mappings from direct products of bundles to bundles. Nevertheless, in going through this section, it would be worthwhile for the reader to give special attention to the case where the domain is a single bundle-and not a product.

Let $X_{1}, \ldots, X_{n}, Y$ be locally compact Hausdorff spaces, $\left\{A_{x_{1}}: x_{1} \varepsilon X_{1}\right\}, \ldots$, $\left\{A_{x_{n}}: x_{n} \varepsilon X_{n}\right\},\left\{B_{y}: y \in Y\right\}$ flelds of Banach spaces over these base spaces, $A_{1}, \ldots, A_{n}, B$ the bundle spaces and $\Pi_{1}: A_{1} \rightarrow X_{1}, \ldots, \Pi_{n}: A_{n} \rightarrow X_{n}, \tau: B \rightarrow Y$ the projections. Let $(A, \pi)$ denote the product bundle over $x=x_{1} \times \ldots \times x_{n}$ given by $A=A_{1} \times \ldots \times A_{n}$ and $\pi=\pi_{1} \times \ldots \times \pi_{n} \cdot($ Recall [11].) In this context, an (abstract) bundle morphism $(\phi, *)$ from the product $(A, \pi)$ into $(B, \tau)$ will be a pair of mappings

$$
\phi: A \rightarrow B, \quad \psi: X \rightarrow Y
$$

satisfying $\tau \phi=\psi \pi$. Thus, such $\phi$ is fiber preserving in the sense that $\phi\left(A_{X}\right) \subseteq B_{\psi(x)}, x \in X$, where $x=\left(x_{1}, \ldots, x_{n}\right)$ and $A_{x}=A_{x_{1}} x_{\ldots} \ldots A_{x_{n}}$. Note that we are not requiring that $\phi$ or $\psi$ be continuous. In fact, our objective here is to develop the notions of continuity and measurability for $(\phi, \psi)$.

Clearly, a bundle morphism $(\phi, \psi)$ may be viewed as a pair $(\Phi, \psi)$ consisting of a mapping $\psi: X \rightarrow Y$ together with a family of mappings $\Phi=\left\{\Phi_{x}: x \in X\right\}$, satisfying $\Phi_{x}\left(A_{x}\right) \subseteq B_{\psi(x)}, x \in X$. The connection between $\Phi$ and $\phi$ is then given by

$$
\begin{aligned}
\phi(a) & =\Phi_{\pi(a)}(a), a \varepsilon A, \\
\text { 1.e. } & \Phi_{x}=\phi \mid A_{X}, x \varepsilon X .
\end{aligned}
$$


DEFINITION 3.1. A Banach bundle morphism from $(A, \pi)$ into $(B, \tau)$ 1s a bundle morphism $(\phi, \psi)$ satisfying

$$
\Phi_{x} \varepsilon \operatorname{Hom}^{\mathrm{n}}\left(\mathrm{A}_{\mathrm{x}}, \mathrm{B}_{\psi(\mathrm{x})}\right), \mathrm{x} \varepsilon \mathrm{X}
$$

(See the Appendix for the definition and required properties of such spaces of bounded n-1inear mappings.) Thus, $\Phi$ may be viewed as a vector field in the product

$$
\Pi_{x} \operatorname{Hom}^{n}\left(A_{x}, B_{\psi(x)}\right)
$$

Now let $D_{1} \subseteq x_{1}, f_{1} \in \Pi_{D_{1}} A_{x_{1}}, 1=1 \leq n$, with $D=D_{1} x_{\ldots} \ldots D_{n}$ and $f=\left(f_{1}, \ldots, f_{n}\right)$. Then $\Phi$ defines a mapping $\Phi f: D+B$ given by

$$
\begin{aligned}
\phi f(x) & =\Phi_{x}(f(x))=\Phi_{x_{1}, \ldots, x_{n}}\left(f_{1}\left(x_{1}\right), \ldots, f_{n}\left(x_{n}\right)\right) \\
& =\phi(f(x)) \\
& =\phi\left(f_{1}\left(x_{1}\right), \ldots, f_{n}\left(x_{n}\right)\right), x \in D .
\end{aligned}
$$

Clearly, $\Phi f=\phi \cdot f$. Moreover, since $f_{1}\left(x_{1}\right) \varepsilon A_{x_{1}}, 1 \leq 1 \leq n$, we have $f(x) \varepsilon A_{x}$, so that $\Phi f(x) \varepsilon B_{\psi(x)}, x \in D$. Thus, $\Phi f \in \Pi_{D} B_{\psi(x)}$, 1.e.

$$
\Phi: \Pi_{1} \Pi_{D_{1}} A_{1}+\Pi_{D} B_{\psi(x)}
$$

However, $\Pi_{1} \Pi_{D_{1}} A_{x_{1}}$ may be vlewed as $\Pi_{D} A_{x}, 1 . e$.

$$
\Phi: \Pi_{D} A_{x} \rightarrow \Pi_{D} B_{\psi(x)}
$$

Next suppose that $\Lambda_{1}, \ldots, \Lambda_{n}, \Delta$ are continuity structures in the spaces $\pi A_{x_{1}}, \ldots, \pi A_{x_{n}}, \Pi B_{y}$. If $\psi$ is continuous, then 1 induces a continulty structure $\Delta^{\psi}$ in $\pi B_{\psi(x)}$ as in section 2. On the other side, we have the product $\Lambda=\Lambda_{1} \times \ldots \times \Lambda_{n}$ of the continulty structures $\Lambda_{1}, \ldots, \Lambda_{n}$ in $\pi A_{x_{1}} \times \ldots \times \pi_{x_{n}}=\Pi_{x} A_{x}$ DEFINITION 3.2. Let $(\phi, \psi)$ be as in 3.1 with aEA. Then $(\phi, \psi)$ is continuous at a (relative to $\Lambda, \Delta$ ) if $\phi$ is continuous at a and $\psi$ is continuous at $\pi(a)$.

DEFINITION 3.3. The operator field $\Phi$ is continous at $x$ in $x$ (relative to $\Lambda, \Delta$ ) if, for each $h$ in $\Lambda$, the mapping $\phi h=\phi \cdot h$ is continuous at $x$. The field $\Phi$ is locally bounded at $x$ in $x$ 1f, for each compact subset $k$ of $x$ containing $x$, the set 


$$
\left\{\left\|\Phi_{\tau}\right\|: \tau \varepsilon K\right\}
$$

of operator norms is bounded in $\mathrm{R}$.

LEMMA 3.4. (1) If $\Phi$ is continuous at $x$, then $\psi$ is continuous at $x$. (2) If $\psi$ is continuous on $x$, then $\Phi$ is continuous at $x$ in $x$ if and only if, for each $h$ in $\Lambda$, the vector field $\Phi$ in $\Pi B_{\psi(t)}$ is $\Delta^{\psi}$-continuous at $x$ PROOF. (1) If $x_{j} \rightarrow x$ in $x$, the zero element $\theta=\left(\theta_{i}\right)$ of $\Lambda$ satisfies: $\theta_{x_{j}} \rightarrow \theta_{x} \cdot$ (2) This follows from 2.2.

For $\mathrm{f}_{i} \varepsilon \Pi_{\mathbf{x}_{i}}$, let

$$
\left\|\mathrm{f}_{i}\right\|_{X_{i}}=\sup \left\{\left\|\mathrm{f}_{i}\left(\mathrm{x}_{i}\right)\right\|: x_{i} \varepsilon X_{i}\right\}, \quad 1 \leq i \leq n
$$

PROPOSITION 3.5. For $(\phi, \psi)$ as in 3.2 , we have:

$$
\left\|\Phi_{x}\right\|=\sup \left\{\left\|\Phi_{x}(f(x))\right\|: f=\left(f_{1}, \ldots, f_{n}\right) \varepsilon \Pi_{i} c_{c}\left(\Lambda_{i}\right),\left\|f_{i}\right\|_{X_{i}} \leq 1, i=1, \ldots, n\right\}, x \in X
$$

PROOF. The proof of 9.2 of [8] can be adapted to this proposition.

COROLLARY 3.6. If $\Phi$ is continuous at $x$ in $X$, then it is locally bounded at $x$--assuming $\Lambda_{i}=\mathrm{C}\left(\Lambda_{i}\right), 1 \leq \mathrm{i} \leq \mathrm{n}$.

PROOF. By hypothesis, $\Phi f: X \rightarrow B$ is continuous at $x$ for each $f \varepsilon \Pi_{i} C_{c}\left(\Lambda_{i}\right)$. Hence, the scalar functions $\|\Phi f(\bullet)\|$ are also continuous. This corollary then follows from 3.5, together with the fact that the supremum of continuous functions is lower semi-continuous and hence, locally bounded.

REMARK 3.7. If we were not assuming $\Lambda_{i}=C\left(\Lambda_{i}\right), 1 \leq i \leq n$, then the two versions of continuity for $\Phi$ in 3.3 would be equivalent for locally bounded $\Phi[7$, p.84] This is reasonable in view of 3.6 .

THEOREM 3.8. Let $x \in X$. Then $(\psi, \psi)$ is continuous at each a in $A_{x}$ if and only if $\Phi$ is continuous at $x$ (as in $[1, \mathrm{p} .32]$ ).

COROLLARY 3.9. Let $x_{i} \in D_{i} \underline{c X}_{i}, 1 \leq i \leq n$. Suppose $(\phi, \psi)$ is continuous at each $a$ in $A_{x}$, i.e. $\Phi$ is continuous at $x=\left(x_{1}, \ldots, x_{n}\right)$. If $f_{i}$ in $\Pi_{D_{i}} A_{x_{i}}$ is $\Lambda_{i}$-continuous at $x_{i}$, then for $f=\left(f_{1}, \ldots, f_{n}\right)$, the mapping 


$$
\Phi_{\mathrm{f}}: \mathrm{D}_{1} \times \ldots \times \mathrm{D}_{\mathrm{n}} \rightarrow \mathrm{B}
$$

is continuous at $x$ for the $\Delta$-topology of $B$.

Next we develop measurability for $(\phi, \psi)$ and $\Phi$. Let $\mu_{i}$ be a positive Radon measure on $x_{i}, 1 \leq i \leq n$. Then $\mu=\mu_{1} \ldots \ldots \mu_{n}$ is a positive Radon measure on $\mathrm{X}[10]$.

DEFINITION 3.10. The Banach bundle morphism $(\phi, \psi)$ (resp. operator field $\Phi$ ) is measurable (relative to $\Lambda, \Delta, \mu$ ) if, for each $h$ in $\Lambda$, the mapping $\phi h$ (resp. $\Phi \mathrm{h}$ ) of $\mathrm{X}$ into $\mathrm{B}$ is measurable. Thus, $(\phi, \psi)$ is measurable if and only if $\Phi$ is measurable (recall 3.8).

LEMMA 3.11. (1) If $\Phi$ is measurable, then $\psi$ is measurable (recal1 3.4). (2) If $\psi$ is continuous, then $\Phi$ is measurable if and only if, for each $h$ in $\Lambda$, the vector field $\Phi_{h}$ in $\Pi_{\psi(x)}$ is $\left(\Delta^{\psi}, \mu\right)$-measurable (recall 2.2).

There is a stronger notion of measurability for $\Phi$ and $(\phi, \psi)$ (as in $[8, \S 10]$ ) which is useful for our needs.

DEFINITION 3.12. The Banach bundle morphism $(\phi, \psi)$ (resp. operator field $\Phi$ ) is ultra-measurable if, for each compact subset $K$ of $X$ and $\varepsilon>0$, there exists a compact subset $K_{\varepsilon}$ of $\mathrm{K}$ such that $\mu\left(\mathrm{K}-\mathrm{K}_{\varepsilon}\right)<\varepsilon$ and $(\phi, \dot{\psi})$ (resp. $\left.\Phi\right)$ is continuous on $\mathrm{K}_{\varepsilon}$. Thus, $(\phi, \psi)$ is ultra-measurable if and only if $\Phi$ is.

THEOREM 3.13. If $\Phi$ is ultra-measurable, then it is measurable. The converse is true if, in addition, $\Phi$ is locally bounded and each $\Lambda_{1}$ is countably dense, $1 \leq \mathrm{i} \leq \mathrm{n}$

PROOF. This is proved as in Prop. 20 of [7].

THEOREM 3.14. Suppose $\Phi$ is measurable. If $f=\left(f_{1}, \ldots, f_{n}\right)$ is an element of $\Pi_{i} M\left(\Lambda_{i}, \mu_{i}\right)$, then $\operatorname{\Phi f} \varepsilon M(X, B, \mu)$.

REMARK 3.15. Once again, if we were not assuming $\Lambda_{i}=c\left(\Lambda_{i}\right), 1 \leq i \leq n$, then there would be two versions of measurability for $\Phi$ given in 3.10. However, in view of 3.14 , these would be equivalent.

REMARK 3.16. As we indicated at the beginning of this seciton, the case $n=1$ 
1s of special interest. Specifically, for this case, this section contains the definttions and properties of continuous, measurable, and ultra-measurable Banach bundle morphisms.

\section{PRODUCT FIELDS.}

Having established the foundations for our analytical needs, we turn to our algebraic needs--namely multiplication (1.e. convolution) and involution. In this section we develop multiplication in $A$ by means of a product fleld of operators.

Suppose $G$ is a locally compact group and $\left\{A_{x}: x \in G\right\}$ is a fleld of Banach spaces over $G$ with $A$ as before. Then $\operatorname{Hom}^{2}\left(A_{x} \times A_{y}, A_{x y}\right)$ is the Banach space (A3) of bounded bilinear operators from $A_{x} \times A_{y}$ into $A_{x y},(x, y) \varepsilon G \times G$. Consequent$1 y$,

$$
\left.\left\{\operatorname{Hom}^{2} A_{x} x A_{y}, A_{x y}\right):(x, y) \varepsilon G \times G\right\}
$$

Is a field of Banach spaces over GXG and

$$
\pi \operatorname{Hom}^{2}\left(A_{x} x A_{y}, A_{x y}\right)
$$

Is a linear space of operator flelds. If $P$ is such a fleld, then for $(x, y) \varepsilon G \times G$, the mapping

$$
P_{x, y}: A_{x} \times A_{y} \rightarrow A
$$

is a bounded bilinear operator with range contained in $A_{x y}$. Let the field of 1dentity operators on the $\left\{A_{x}: x \in G\right\}$ be denoted by $\left\{E_{x}: x \in G\right\}$. (This is in $\Pi_{G} \operatorname{Hom}\left(A_{x}\right)$

DEFINITION 4.1. Let $P E \Pi H^{2}{ }^{2}\left(A_{x} \times A_{y}, A_{x y}\right)$. Then $P$ is a product (or multiplication or convolution) field if $\left\|P_{x, y}\right\| \leq 1$ and

$$
P_{x y, z}\left(P_{x, y} \times E_{z}\right)=P_{x, y z}\left(E_{x} \times P_{y, z}\right), x, y, z \varepsilon G
$$

For conventence, let $P=\Pi H H^{2}\left(A_{x} \times A_{y}, A_{x y}\right)$. Note that an element of $P$ is not necessarily a product field. Moreover, let $p$ denote the mapping of $A \times A \rightarrow A$ defined by

$$
p(a, b)=P_{\pi(a), \pi(b)}(a, b), a, b \varepsilon A
$$

Since the mapping $\psi: G \times G \rightarrow G$ given by group multiplication satisfies 
$\psi(\pi \times \pi)=\pi p$, It follows that $(p, \psi)$ is a Banach bundle morphism of (AxA, $\pi \pi)$ into $(A, \pi)$. Furthermore, since $\psi$ is continuous, if $\Lambda$ is a continuity structure in $\Pi_{x}$, then we obtain the induced continuity structure $\Lambda^{\psi}$ in

$$
\pi A_{x, y} \stackrel{\text { def. }}{=} \pi A_{\psi(x, y)}=\pi A_{x y}
$$

as In Section 2. We w1ll denote $\Lambda^{\psi}$ by $\Lambda^{\prime}, A^{\psi}$ by $A^{\prime}$ and $\pi^{\psi}$ by $\pi^{\prime}$.

We w11l also require that product fields be measurable relative to $\Lambda$ and (right) Haar measure $\mu$ on $G$ as in Section 3 . It w111 be instructive to summarize the results of Section 3 for $(p, P, \psi)$.

THEOREM 4.2. Let $P$ be an element of $P$ with $p$ as above. Then the following are equivalent for $\left(x_{0}, y_{0}\right) \in G \times G$ :

(1) $P$ is continuous at $\left(x_{0}, y_{0}\right)$.

(11) For each $h, k$ in $\Lambda$, the mapping $P(h, k): G \times G \rightarrow A$ (as in 53) 1s continuous at $\left(x_{0}, y_{0}\right)$.

(111) For each $h, k$ in $\Lambda$, the vector field $P(h, k)$ in $\Pi_{x y}$ is $\Lambda^{\prime}$-continuous at $\left(x_{0}, y_{0}\right)$.

(1v) For each $h, k$ in $\Lambda$ the cross-section $P(h, k)$ in $S\left(G \times G, A^{\prime}\right)$ is continuous at $\left(\mathrm{x}_{0}, \mathrm{y}_{0}\right)$.

(v) The bundle morphism $(p, \psi)$ is continuous at each point $(a, b)$ in $A_{x_{0}} \times A_{y_{0}}$. (v1) The mapping $p$ is continuous at each point $(a, b)$ in $A_{x_{0}} \times_{y_{0}}$. PROOF. Combine $1.8,3.2,3.3,3.4$, and 3.8 .

PROPOSITION 4.3. Let $D_{1}, D_{2} \subseteq G, x_{0} \varepsilon D_{1}, y_{0} \varepsilon D_{2} \cdot$ Suppose $P$ is continunus at $\left(x_{0}, y_{0}\right)$. If $f \varepsilon \Pi_{D_{1}} A_{x}, g \varepsilon \Pi_{D_{2}} A_{y}$ are $\Lambda$-continuous at $x_{0}, y_{0}$ respectively, then the mapping $P(f, 8): D_{1} x_{2} \rightarrow A$ is continuous at $\left(x_{0}, y_{0}\right)$ (resp. $\Lambda^{\prime}$-continuous at $\left.\left(x_{0}, y_{0}\right)\right)$

PROOF. Recall 2.2, 3.9.

THEOREM 4.4. Let $P E P$. Then the following are equivalent:

(1) P is measurable.

(11) For each $h, k$ in $\Lambda$, the mapping $P(h, k): G \times G \rightarrow A$ is measurable. 
(iim) For each $h, k$ in $\Lambda$, the vector field $\left(E_{G \times G}, P(h, k)\right)$ in $\prod_{x y}$ is $\left(\Lambda^{\prime}, \mu \mathrm{\Omega} \mu\right)$-measurable.

(iv) For each $h, k$ in $\Lambda$, the cross-section $\left(E_{G \times G}, P(h, k)\right)$ is measurable relative to $\Lambda^{\prime}$ and $\mu \Omega \mu$.

PROOF. Combine $3.10,3.11$, and 1.12 .

THEOREM 4.5. Let PEP. Then the following are equivalent:

(i) $P$ is ultra-measurable.

(ii) For each compact subset $K$ of $G \times G$ and $\varepsilon>0$, there exists a compact subset $K_{\varepsilon}$ of $K$ such that $\mu \rho\left(K-K_{\varepsilon}\right)<\varepsilon$ and $P$ is continuous on $K_{\varepsilon}$.

If $P$ is a product field and $\Lambda$ is countably dense, then the previous are equivalent to:

(iii) $P$ is measurable.

PROOF. Combine $3.12,3.13$, and 4.1 .

PROPOSITION 4.6. Suppose $P$ in $P$ is measurable and $f, g \in M(\Lambda, \mu)$. Then $P(f, g) \varepsilon M(G \times G, A, \mu \Omega \mu)$

PROOF. Recal1 3.14

\section{INVOLUTION FIELDS .}

The other algebraic operation we need is involution, which will also be introduced by means of a field of operators. Of course, such fields will have to be suitably compatible with product fields in order that the resulting operations yield a *-algebra structure in $A$.

For each $x$ in $G$, let $A_{x}^{-}$denote the Banach space conjugate to $A_{x}$, 1.e. $A_{x}^{-}$and $A_{x}$ are identical except for scalar multiplication which is given in $A_{x}^{-}$by:

$$
\alpha \cdot \mathrm{a}=\bar{\alpha} \mathbf{a}, \alpha \varepsilon \mathbb{\mathbb { C }},{\mathrm{a} \varepsilon \mathrm{A}_{\mathrm{x}}}
$$

Consider the fields $\left\{A_{x}^{-}: x \in G\right\},\left\{A_{x-1}: x \in G\right\}$ of Banach spaces over $G$. The spaces $\left\{\operatorname{Hom}\left(A_{x}^{-}, A_{x-1}\right)\right\}$ form a field of Banach spaces over $G$ and $\Pi H o m\left(A_{x}^{-}, A_{x^{-1}}\right)$ is a linear space of operator fields. Note that the linear mappings from $A_{x}^{-}$into $A_{x^{-1}}$ 
are precisely the conjugate linear mappings from $A_{x}$ into $A_{x}-1$. Thus, if $I$ is an element of $\Pi \operatorname{Hom}\left(A_{x}^{-}, A_{x^{-1}}\right)$, then, for each $x$ in $G$, the mapping

$$
\left.I_{x}: A_{x}^{-} \rightarrow A \text { (resp. } I_{x}: A_{x} \rightarrow A\right)
$$

is bounded and linear (resp. conjugate linear) with range contained in $A_{x-1}$. For convenience, let

$$
\mathrm{S}_{\mathrm{x}, \mathrm{y}}: \mathrm{A}_{\mathrm{x}} \times \mathrm{A}_{\mathrm{y}} \rightarrow \mathrm{A}_{\mathrm{y}} \times \mathrm{A}_{\mathrm{x}}
$$

denote the switching mapping $(a, b) \rightarrow(b, a), a \varepsilon A_{x}, b \varepsilon A_{y}, x, y \varepsilon G$.

DEFINITION 5.1. Let $I$ be an element of $\operatorname{IHom}\left(A_{x^{-}}^{-}, A_{x-1}\right)$. Then $I$ is an $\underline{\text { involution field }}$ if $\left\|I_{x}\right\|=1, I_{x^{-1}}=I_{x}^{-1}$ and

$$
I_{x y} P_{x, y}=P_{y-1, x^{-1}}\left(I_{y} x_{x}\right) S_{x, y}, x, y \varepsilon G
$$

for each product field $P$ in $P$. For convenience, let

$$
I=\Pi \operatorname{Hom}\left(\mathrm{A}_{\mathrm{x}}^{-}, \mathrm{A}_{\mathrm{x}-1}\right) \text {. }
$$

Note that the elements of $I$ are not necessarily involution fields.

For the purposes of this section, let $\psi: G \rightarrow G$ be the inversion homeomorphism $\psi(x)=x^{-1}, x \in G$. If $f$ is a mapping defined on $G$, it is customary to write $\mathrm{f}^{\mathrm{V}}$ for $\mathrm{f}_{\psi}$. Consequently, the induced continuity structure $\Lambda^{\psi}$ in $\Pi_{\psi(\mathrm{x})}=\operatorname{IIA} \mathrm{A}_{\mathrm{x}-1}$ will be denoted by $\Lambda^{\mathrm{v}}$, i.e.

$$
\Lambda^{\mathrm{v}}=\left\{\mathrm{h}^{\mathrm{v}}: \mathrm{h} \varepsilon \Lambda\right\}
$$

Also, denote by $\Lambda^{-}$the continuity structure $\Lambda$ viewed as being in $\Pi_{x}^{-}=\Pi_{x}$. Hence, for $\alpha \varepsilon \mathbb{C}$ and $h^{-} \varepsilon \Lambda^{-}$, we have

$$
\left(\alpha h^{-}\right)(x)=\bar{\alpha} h(x), x \in G \text {. }
$$

Now let $i$ denote the mapping of $A$ into itself given by

$$
i(a)=I_{\pi(a)}(a), a \in A \text {. }
$$

Then $(i, \psi)$ is a bundle morphism of $\left(A^{-}, \pi^{-}\right)$into $\left(A^{v}, \pi^{v}\right)$ where:

(i) $A^{-}=A=U A_{X}^{-}$(disjoint), with conjugate scalar multiplication in the fibers.

(ii) $A^{V}=A=U_{x^{-1}}$ (disjoint), with $A_{x}^{-1}$ the fiber over $x$ in $G$. 
(iii)

$$
\pi^{-}(a)=\pi(a)=x \text {, for } a^{\varepsilon} A_{x}^{-}=A_{x}, x^{\varepsilon G} \text {. }
$$

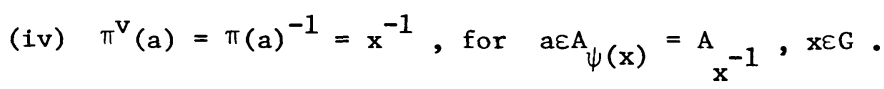

As in the previous section, an involution field will be required to be measurable relative to $\Lambda$ and $\mu$. Here also, it will be instructive to summarize Section 3 for $(i, I, \psi)$.

THEOREM 5.2. Let $I \varepsilon I$ with $i$ as above. Then the following are equivalent for $x_{0}$ in $G:$

(i) I is continuous at $x_{0}$.

(ii) For each $h$ in $\Lambda$, the mapping $I\left(h^{-}\right): G \rightarrow A$ is continuous at $x_{0}$.

(iii) For each $h$ in $\Lambda$, the vector field $I\left(h^{-}\right)$in $\pi A x^{-1}$ is $\Lambda^{\mathrm{v}}$-continuous at $\mathrm{x}_{\mathrm{o}}$.

(iv) For each $h$ in $\Lambda$, the cross-section $I\left(h^{-}\right)$in $S\left(G, A^{v}\right)$ is continuous at $x_{0} \cdot$

(v) The bundle morphism $(i, \psi)$ is continuous at each point a in $A_{x_{0}^{-}}^{-}$.

(vi) The mapping $i$ is continuous at each point a in $A_{x_{0}^{-}}^{-}$.

PROOF. Combine $1.8,3.2,3.3,3.4$, Rand 3.8 .

PROPOSITION 5.3. Let $D \mathcal{E}, x \in D$. Suppose $I$ in $I$ is continuous at $x$.

If $f \in \Pi A_{y}$ is $\Lambda$-continuous at $x$, then the mapping $I\left(f^{-}\right): D \rightarrow A$ is continuous at

$\mathbf{x}$ (resp. $\Lambda^{\mathrm{v}}$-continuous at $\mathrm{x}$ ).

PROOF. Recall 2.2 and 3.9.

THEOREM 5.4. Let IEI. Then the following are euqivalent:

(i) I is measurable.

(ii) For each $h$ in $\Lambda$, the mapping $I\left(h^{-}\right): G \rightarrow A$ is measurable.

(iii) For each $h$ in $\Lambda$, the vector field $I\left(h^{-}\right)$in $\pi A x^{-1}$ is $\left(\Lambda^{v}, \mu\right)$-measurable.

(iv) For each $h$ in $\Lambda$, the cross-section $\mathrm{Ih}^{-}: G \rightarrow A^{V}$ is measurable realtive to $\left(\Lambda^{\mathrm{v}}, \mu\right)$.

PROOF. Recall 1.12, 3.10, and 3.11.

THEOREM 5.5. Let IEI. Then the following are equivalent: 
(i) I is ultra-measurable.

(ii) For each compact subset $K$ of $G$ and $\varepsilon>0$, there exists a compact subset $K_{\varepsilon}$ of $K$ such that $\mu\left(K-K_{\varepsilon}\right)<\varepsilon$ and $I$ is continuous on $K_{\varepsilon}$ (resp. $i$ is continuous on $\left.\left(\pi^{-}\right)^{-1}\left(K_{\varepsilon}\right)=\pi^{-1}\left(K_{\varepsilon}\right)\right)$.

If $I$ is an involution field and $\Lambda$ is countably dense, then the previous are equivalent to:

(iii) I is measurable.

PROOF. Combine $3.12,3.13$, and 5.1 .

PROPOSITION 5.6. Suppose $I$ is measurable and $f \varepsilon M(\Lambda, \mu)$. Then $I\left(f^{-}\right) \varepsilon M(G, A, \mu)$

PROOF. Recal1 3.14.

\section{GROUP ALGEBRA BUNDLES .}

We are now ready to construct a measurable analogue of Fell's Banach *-algebraic bundle [1]. Let $\left\{A_{x}: x \in G\right\}$ once again be a field of Banach spaces over the locally compact group $G$, with $\Lambda, A, \pi, \mu$ as above. Suppose also that $P$ is a product field in $P$ with $P$ the corresponding product bundle mapping. To introduce multip1ication into $A$, define

$$
a \cdot b=p(a, b)=P_{\pi(a), \pi(b)}(a, b), a, b \varepsilon A
$$

PROPOSITION 6.1. We have:

(i) $a \cdot b \varepsilon A_{x y}$, for $a \varepsilon A_{x}, b \varepsilon A_{y}, i \cdot e ., A_{x} \cdot A_{y} c_{x y}, x, y \varepsilon G$.

(ii) For each $x, y \in G$, the mapping $(a, b)+a \cdot b$ of $A_{x} \times A_{y}$ into $A_{x y}$ is bilinear.

(iii) $(a \cdot b) \cdot c=a \cdot(b \cdot c), a, b, c \varepsilon A$.

(iv) $\|\mathrm{a} \cdot \mathrm{b}\| \leq\|\mathrm{a}\| \cdot\|\mathrm{b}\|$, $\mathrm{a}, \mathrm{b} \varepsilon A$.

To obtain continuity for the product mapping $p: A \times A \rightarrow A$, recall 4.1:

PROPOSITION 6.2. The product in $A$ is continuous if and only if $P$ is continuous.

One of our goals is to generalize the above to the case where the convolution is just measurable--and not necessarily continuous. In this regard, we have:

PROPOSITION 6.3. The product in $A$ is measurable if and only if $P$ is 
measurable. It is ultra-measurable if and only if $P$ is ultra-measurable. If $\Lambda$ is countably dense, then these are equivalent to measurability for $P(4.5)$.

To introduce involution into $A$, suppose further that $I$ is an involution field in $I$ with $i$ as before. For a in $A$, define

$$
a *=i(a)=I_{\pi(a)}(a)
$$

PROPOSITION 6.4. We have:

(i) $\pi(a *)=\pi(a)^{-1}=\pi^{v}(a), a \varepsilon A, i . e .\left(A_{x}\right) * \subseteq A_{x^{-1}}, x \in G$.

(ii) For each $x$ in $G$, the mapping $a \rightarrow a^{*}$ from $A_{x}$ into $A_{x^{-1}}$ (resp. $A_{x}^{-}$ into $A_{x^{-1}}$ ) is conjugate linear (resp. linear).

(iii) $(a \cdot b) *=b * \cdot a *, a, b \varepsilon A$.

(iv) $a * *=a, a \notin A$.

To obtain continuity and measurability for the involution mapping $i: A \rightarrow A$, recal1 5.1 and 5.4 :

PROPOSITION 6.5. The involution in $A$ is continuous if and only if $I$ is continuous.

PROPOSITION 6.6. The involution in $A$ is measurable if and only if $I$ is measurable. It is ultra-measurable if and only if $I$ is. If $\Lambda$ is countably dense, then these are equivalent to the measurability of $I$.

DEFINITION 6.7. By a (measurable) Fell bundle $(A, \pi ; P, I)$ over $G$, we will mean a Banach bundle $(A, \pi)$ over $G$ together with measurable product and involution fields $P$ and $I$. (The underlying continuity structure $\Lambda$ will be understood to be $\operatorname{CS}(G, A)$ as before.) If $P$ and $I$ are continuous, we will say that $(A, \pi ; P, I)$ is a continuous Fell bundle over $G$ (equivalently, a Banach *-algebraic bundle over G ).

In Section 3 of [1], Fell defines a continuous Fell bundle $(A, \pi: \bullet, *)$ over $G$ to be a Banach bundle $(A, \pi)$ over $G$ with product - and involution * given axiomatically. Clearly, the corresponding product and involution fields $P$ and $I$ are then determined as follows: 


$$
\begin{aligned}
& P_{x, y}(a, b)=a \cdot b, \\
& I_{x}(a)=a^{*}, \quad a \varepsilon A_{x}, b \varepsilon A_{y}, x, y \in G .
\end{aligned}
$$

Of course, these fields are continuous relative to $\Lambda=\operatorname{CS}(G, A)$, since $\cdot$ and * are continuous operations on $A(6.2,6.5)$. To extend his construction to that of a measurable Fell bundle, the appropriate measurability requirements on $\cdot$ and * would be given by 4.4 and 5.4 .

EXAMPLE $6.8[2, \mathrm{p} .130]$. Let $\mathrm{B}$ be the 2-dimensional Banach *-algebra $\boldsymbol{c}^{2}$ with *-algebra structure given by

$$
\begin{aligned}
& (a, b)\left(a^{\prime}, b^{\prime}\right)=\left(a a^{\prime}, b b^{\prime}\right), \\
& (a, b) *=\left(b^{-}, a^{-}\right)
\end{aligned}
$$

and norm given by

$$
\|(a, b)\|=\max (|a|,|b|), a, a^{\prime}, b, b^{\prime} \varepsilon \mathbb{C} \text {. }
$$

Let $G$ be the cyclic group $\{1,-1\}$ of order 2 and $\left\{A_{1}, A_{-1}\right\}$ the closed subspaces of $B$ given by

$$
\begin{aligned}
A_{1} & =\{(a, a): a \varepsilon \mathbb{C}\}, \\
A_{-1} & =\{(a,-a): a \varepsilon \mathbb{C}\} .
\end{aligned}
$$

Since $G$ is discrete, we have $\Lambda=A_{1} \times A_{-1} \cdot$ Define $P$ and $I$ by

$$
\begin{aligned}
& P_{x, y}((a, x a),(b, y b))=(a b, x y a b), \\
& I_{x}((a, x a))=\left(a^{-}, x a^{-}\right), x, y \varepsilon G, a \varepsilon A_{x}, b \varepsilon A_{y} .
\end{aligned}
$$

Of course, $P$ and $I$ are continuous.

EXAMPLE $6.9[2, \mathrm{P} .131]$. Let $\mathrm{D}=\{\mathrm{z} \varepsilon \mathbb{C}:|z| \leq 1\}$ and $\partial \mathrm{D}$ the ususal boundary of D . Let $B$ be the Banach *-algebra C(D) with pointwise *-algebra structure. For $G=\{1,-1\}$, let $A_{1}, A_{-1}$ be the closed subspaces of $B$ given by

$$
\begin{gathered}
A_{1}=\{f \varepsilon C(D): f(-z)=f(z), z \varepsilon \partial D\}, \\
A_{-1}=\{f \varepsilon C(D): f(-z)=f(z), z \varepsilon \partial D\} .
\end{gathered}
$$

Then $\Lambda=A_{1} \times A_{-1}$ once again and continuous $P$ and $I$ are given by 


$$
\begin{aligned}
& P_{x, y}(f, g)=x y(f \cdot g), \\
& I_{x}(f)=f^{-}, x, y \varepsilon G, f A_{x}, g \varepsilon_{y} .
\end{aligned}
$$

Before leaving this section, we wish to point out one of the main consequences of replacing a continuous Fell bundle by a measurable one.

DEFINITION $6.10[2, \mathrm{p} .115]$. A measurable Fell bundle $(A, \pi: P, I)$ over $G$ is saturated if the span of $A_{x} \cdot A_{y}$ is dense in $A_{x y}$, i.e. the range of $P_{x, y}$ has dense span in $A_{x y}$, for all $x, y \in G$.

PROPOSITION $6.11[2, \mathrm{P} .115]$. If $(A, \pi ; P, I)$ is saturated, then:

(i) The span of $A_{e} \cdot A_{x}$ is dense in $A_{x}, x \in G$.

(ii) The span of $A_{x} \cdot A_{x-1}$ is dense in $A_{e}, x \in G$.

Conversely, if $P$ is continuous, then ( $i$ ) and ( $i i)$ imply saturation.

Hence, the converse implication is questionable for measurable Fell bundles since its proof depends on the continuity of the product in $A$. This also affects Propositions 11.4 and 11.5 of [2] in the same way.

\section{BUNDLE IDENTITIES.}

The purpose of this section is to extend (and rephrase) Fell's study of approximate identities (units) in $[1,2]$ to the context of a measurable Fell bundle.

Let $(A, \pi ; P, I)$ be such a bundle. It is clear then that the fiber $A_{e}$ over the identity $e$ in $G$ is a Banach *-algebra.

DEFINITION $7.1[1, \mathrm{p} .34]$. A (bounded) $\underline{\text { approximate }} \underline{\text { identity }}$ in $A$ is a net $\left\{u_{j}\right\}$ in $A_{e}$ satisfying:

(i) $\left\|u_{j}\right\| \leq \beta$, all $j$, for some $\beta>0$.

(ii) $\left\|u_{j} \cdot a-a\right\| \rightarrow 0, a \varepsilon A$.

(iii) $\left\|a \cdot u_{j}-a\right\| \rightarrow 0, a \varepsilon A$.

In particular, $\left\{u_{j}\right\}$ is an approximate identity in $A_{e}$ in the usual sense.

DEFINITION 7.2 [1,p.34]. The net $\left\{u_{j}\right\}$ in $A_{e}$ is a strong approximate identity if it is an approximate identity and (ii) and (iii) of 7.1 hold uniformly 
on compact subsets of $A$.

In Prop. 11.1 of [2], Fell showed that these two notions agree in the case of continuous Fell bundles. This appears to be false in the measurable case. To determine what is true, we proceed as follows.

Let $D \subseteq G, f \in \Pi_{D} A_{x}$ and $a \varepsilon A_{e}$. The left and right translates of $f$ by a are defined by

$$
a^{f(x)}=a \cdot f(x)=P_{e, x}(a, f(x))
$$

and

$$
f_{a}(x)=f(x) \cdot a=P_{x, e}(f(x), a), x \in D .
$$

Then $a^{f}, f_{a}$ belong to $\Pi_{D} A$ which is a linear space, i.e. $\Pi_{D} A_{x}$ is a (twosided) $A_{e}$-module in general. Now consider $C(D, \Lambda) \subseteq \Pi_{D} A x \cdot$

PROPOSITION 7.3. Let $\Gamma$ be a total subset of $C(\Lambda)$. Then the following are equivalent:

(i) $C(D, \Lambda)$ is a left (resp. right) $A_{e}$-module.

(ii) For each $h$ in $\Gamma$, a in $A_{e}$, the vector field $a^{h}\left(\right.$ resp. $\left.h_{a}\right)$ is $\Lambda$-continuous on $\mathrm{D}$.

DEFINITION 7.4. We say that $C(\Lambda)$ is locally an $A_{e}$-module if $C(K, \Lambda)$ is an

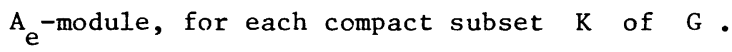

The following is our measurable Fell bundle version of Prop. 11.1 of [2] .

THEOREM 7.5. Let $\left\{u_{j}\right\}$ be an approximate identity in $A$. If $C(\Lambda)$ is locally an $A_{e}$-module, then $\left\{u_{j}\right\}$ is a strong approximate identity in $A$. In particular, if multiplication in $A$ is continuous, then $C(\Lambda)$ is actually (globally) and $\mathrm{A}_{\mathrm{e}}$-module.

REMAR: 7.6. Observe that if $C(\Lambda)$ is locally an $\mathrm{e}_{\mathrm{e}}$-module, then the corollaries 11.2 and 11.3 of [2] are valid.

\section{MULTIPLIERS.}

There are two additional significant distinctions between continuous and measurable Fell bundles which involve multipliers. We will briefly discuss here the multiplier theory $[1, \S 4]$ we require in this and succeeding sections. 
DEFINITION 8.1. If $x$ is an element of $G$, then a multiplier $m$ of order $x$ for the measurable Fell bundle $(A, \pi ; P, I)$ is a pair $\left(m_{1}, m_{2}\right)$ satisfying the following :

(i) $\mathrm{m}_{1}, \mathrm{~m}_{2}$ are continuous mappings of $A$ into itself which are bounded in the sense that

$$
\left\|m_{i}\right\|=\sup \left\{\left\|_{m_{i}}(a)\right\|: a \varepsilon A\right\}
$$

is finite for $i=1,2$.

(ii) For each $y \varepsilon G, m_{1}$ (resp. $m_{2}$ ) is a linear mapping of $A_{y}$ into $A_{x y}$ $\left(\right.$ resp. $\left.A_{y x}\right)$.

(iii) $a \cdot m_{1}(b)=m_{2}(a) \cdot b, a, b \varepsilon A$

(iv) $m_{1}(a \cdot b)=m_{1}(a) \cdot b, a, b \varepsilon A$

(v) $\mathrm{m}_{2}(\mathrm{a} \cdot \mathrm{b})=\mathrm{a} \cdot \mathrm{m}_{2}(\mathrm{~b}), \mathrm{a}, \mathrm{b} \varepsilon A$

For convenience, as usual, we will write ma for $\mathrm{m}_{1}(\mathrm{a})$ and am for $\mathrm{m}_{2}(\mathrm{a})$, $a \notin A$. Let $M_{x}(A)$ denote the set of multipliers of order $x$ and $M(A)=U_{G} M_{x}(A)$. Also, let $\pi^{0}: M(A) \rightarrow G$ be the canonical projection.

Each $M_{x}(A)$ is a Banach space under the canonical linear operations and norm given by

$$
\|\mathrm{m}\|_{0}=\max \left(\left\|\mathrm{m}_{1}\right\|,\left\|\mathrm{m}_{2}\right\|\right), \operatorname{m\varepsilon M}_{\mathrm{x}}(\mathrm{A}), \mathrm{x} \varepsilon \mathrm{G} \text {. }
$$

Thus, $\left(M(A), \pi^{\circ}\right)$ is algebraically a Banach bundle. Moreover, there is a product and an involution in $M(A)$ given by

$$
\left(m \cdot m^{1}\right) a=m\left(m^{\prime} a\right), a\left(m \cdot m^{\prime}\right)=(a m) m^{\prime}
$$

and

$$
\mathrm{m} * \mathrm{a}=(\mathrm{a} * \mathrm{~m}) *, a \mathrm{~m} *=(\mathrm{ma} *) *
$$

for $a \notin A, m, m^{\prime} \varepsilon M(A)$. The operations have the following properties:

(1) If $\operatorname{m\varepsilon M}_{x}(A), m^{\prime} \varepsilon M_{y}(A)$, then $m^{\prime} \varepsilon M_{x y}(A)$.

(2) The product is bilinear on $M_{x}(A) \times M_{y}(A)$.

(3) The product is associative.

(4) If $\operatorname{m\varepsilon g}_{x}(A)$, then $m * \varepsilon M_{x}-1(A)$.

(5) The involution is conjugate linear on $M_{x}(A)$. 
(6) The involution is anti-multiplicative.

(7) The involution is self-invertible.

(8) $\left\|\mathrm{mm}^{\prime}\right\|_{0} \leq\|\mathrm{m}\|_{0} \cdot\left\|\mathrm{m}^{\prime}\right\|_{0}$ and $\|\mathrm{m} *\|_{0}=\|\mathrm{m}\|_{0}$.

(9) The left and right identity mappings of $A$ form the identity of $M(A)$ in $M_{e}(A)$.

We are now ready to describe one of the distinctions between continuous and measurable Fell bundles referred to at the beginning of this section.

If the Fell bundle $(A, \pi ; P, I)$ is continuous, then $A$ can be mapped into $M(A)$ by right and left multiplication:

$$
m_{b} a=b a, a m_{b}=a b, a, b \varepsilon A \text {. }
$$

However, in the measurable (non-continuous) case, this does not seem possible, since the left and right multiplications may fail to be continuous.

The remaining distinction involves the notion of unitary multiplier.

DEFINITION 8.2. If $m \varepsilon M(A)$, then $m$ is unitary if $\|m\| \leq 1$ and $\mathrm{m} *_{\mathrm{m}}=\mathrm{mm} *=1$, the canonical identity in $M(A)$.

The unitary multipliers $U(A)$ in $M(A)$ form a group under multiplication. Also, if $U_{x}(A)=U(A) \cap M_{x}(A)$, then

$$
U(A)=U_{G} U_{x}(A)
$$

and

$$
\mathrm{m}_{\mathrm{x}}^{\star \varepsilon} \mathrm{U}^{-1}(A) \text {, if } \mathrm{m} \varepsilon \mathrm{U}_{\mathrm{x}}(\mathrm{A}), \mathrm{x} \varepsilon \mathrm{G}
$$

DEFINITION 8.3. A measurable Fell bundle has enough unitary multipliers if, for each $x$ in $G, U_{x}(A) \neq \phi \quad[2, p .122]$.

LEMM1 8.4. If $(A, \pi ; P, I)$ has enough unitary multipliers, then the Banach spaces $\left\{A_{x}: x \in G\right\}$ are all isometrically isomorphic $[2, p .123]$.

LEMMA 8.5. If $P$ is continuous and $A$ has an approximate unit as well as enough unitary multipliers, then $A$ is saturated (6.10 and [2,prop.11.5]).

REMARK 8.6. In general, if $A$ is saturated, then it may not have enough unitary multipliers even if $P$ is continuous and $A$ has an identity [2,p.130]. 
The implication in 8.5 is questionable in the measurable case because its proof, namely Prop. 11.4 of [2], is questionable for non-continuous P . (Recall the end of Section 6.)

There is a notion stronger than "enough unitary multipliers" called "homogeneity" which we will study in detail in Section 10.

Finally, $M(A)$ can be equipped with a topology called the strong topology $[1, \S 5]$. In this topology, a net $\left\{M_{j}\right\}$ in $M(A)$ converges to $m$ in $M(A)$ if

$$
\mathrm{m}_{\mathrm{j}} \mathrm{a} \rightarrow \mathrm{ma}, \mathrm{m}_{\mathrm{j}} \rightarrow \mathrm{am}, \mathrm{a} \varepsilon A
$$

For this topology, involution is continuous and the product is separately continuous. The mapping $\pi^{\circ}: M(A) \rightarrow A$ is continuous relative to this topology but possibly not open. Consequently, $\pi^{O} \mid U(A)$ is a continuous homomorphism of $U(A)$ into $G$ (onto if there exist enough unitary multipliers). Note that multiplication in $U(A)$ is separately continuous but possible not jointly continuous.

$$
\text { Let } \quad M^{1}(A)=\left\{m \varepsilon M(A):\|m\|_{0} \leq 1\right\} .
$$

The following will be useful in Section 10 .

PROPOSITION 8.7. If $A$ has a strong approximate identity and $P$ is ultra measurable, then the mappings $(m, a) \rightarrow$ ma and $(m, a) \rightarrow a m$ of $M^{1}(A) \times A$ into $A$ are measurable in the sense of 3.10. In particular, if $P$ (i.e., p) is continuous, then these mappings are continuous (compare with [1, Prop.5.1]).

\section{GENERALIZED GROUP ALGEBRAS.}

To construct a Banach *-algebra from a measurable Fell bundle $(A, \pi ; P, I)$ over $G$, let $L^{1}(\Lambda, \mu)$ denote the Banach space of (null equivalence classes) of $(\Lambda, \mu)$ measurable vector fields $f$ which are $\mu$-integrable, i.e. for which

$$
\|f\|_{1}=\int_{G}\|f(x)\| d \mu(x)<\infty
$$

(See $[7,8]$ for the details.) The subspace $C_{c}(\Lambda)$ of $c(\Lambda)$ consisting of vector fields with compact support is well-known to be dense in $L^{1}(\Lambda, \mu)$. Also, in view of the results in section $1, L^{1}(\Lambda, \mu)$ is the vector field version of the space $L^{1}(A, \mu)$ of $\mu$-measurable integrable cross-sections $[1, \S 2]$. Let $\delta$ be the (right) modular function for G. Before proceeding further, let us record an important 
result for future use. Once again, let $\lambda: G \times G \rightarrow G$ be the left projection (recall 2.5)

LEMMA 9.1. Let $f \in L^{1}\left(\Lambda^{\lambda}, \mu \Omega \mu\right)$. Then for $\mu-a \cdot a \cdot x$ in $G$, the integral

$$
\int_{G} f(x, y) d \mu(y)
$$

belongs to $A_{x}$ and the resulting ( $\mu-a . e$. defined) vector field

$$
x \rightarrow \int_{G} f(x, y) d \mu(y)
$$

belongs to $\mathrm{L}^{1}(\Lambda, \mu)$.

PROOF. This is the vector field analogue of Prop. 2.11 of [1].

Now, for $f, g$ in $L^{1}(\Lambda, \mu)$, consider the vector field

$$
(x, y) \rightarrow f\left(y^{-1}\right) \cdot g(y x)=P(f, g)\left(y^{-1}, y x\right)
$$

in $\Pi_{\lambda(x, y)}$

PROPOSITION 9.2. This vector field is $\left(\Lambda^{\lambda}, \mu \Omega \mu\right)$-measurable.

LEMMA 9.3. The vector field of 9.2 belongs to $L^{1}\left(\Lambda^{\lambda}, \mu \mu\right)$ and its $L^{1}$-norm is at most $\|\mathrm{f}\|_{1} \cdot\|\mathrm{g}\|_{1}$.

PROOF. This is a straightforward application of the scalar Fubini Theorem.

COROLLARY 9.4. For $f, g$ in $\mathrm{L}^{1}(\Lambda, \mu)$, the vector field

$$
x \rightarrow \int_{G} f\left(y^{-1}\right) \cdot g(y x) d \mu(y)
$$

(defined $\mu$-a.e. on $G$ ) belongs to $L^{1}(\Lambda, \mu)$.

PROOF. Apply 9.1 to the integrand.

As a result of the previous discussion, for $f, g$ in $L^{1}(\Lambda, \mu)$, we may define a vector field $f \cdot g$ in $L^{1}(\Lambda, \mu)$ by

$$
(f \cdot g)(x)=\int_{G} f\left(y^{-1}\right) \cdot g(y x) d \mu(y), \mu-a \cdot a \cdot x \text { in } G \text {. }
$$

We thus obtain a binary operation - (multiplication or convolution) on $\mathrm{L}^{1}(\Lambda, \mu)$ which satisfies

$$
\|\mathrm{f} \cdot \mathrm{g}\|_{1} \leq\|\mathrm{f}\|_{1} \cdot\|\mathrm{g}\|_{1}, \mathrm{f}, \mathrm{g} \varepsilon \mathrm{L}^{1}(\Lambda, \mu)
$$

REMARK 9.5. Before going any further, observe that Fell defines convolution 
first in $C_{c}(\Lambda)\left[1,\{8]\right.$ and then extends it to all of $L^{1}(\Lambda, \mu)$. Note that in his case (that of a continuous Fell bundle), $C_{c}(\Lambda)$ is closed under multiplication as a consequence of the continuity of multiplication in A. However, this is not true for a measurable Fell bundle. Hence, for us, there is less advantage in first multiplying elements of $C_{c}(\Lambda)$, since the products may not be in $C_{c}(\Lambda)$.

PROPOSITION 9.6. Under multiplication, $L^{1}(\Lambda, \mu)$ is a Banach algebra which we denote by $L^{1}(\Lambda, \mu ; P)$.

Next, we define involution in $L^{1}(\Lambda, \mu ; P)$. For $f$ in $\Pi A_{x}$, define $f^{*}$ in $\Pi A_{x}$ by

$$
f *(x)=\delta(x)^{-1} f\left(x^{-1}\right) *=\delta(x)^{-1} I\left(f^{-}\right)(x)=\delta(x)^{-1} I_{x}(f(x)), x \in G
$$

LEMMA 9.7. For each $\mathrm{f}$ in $L^{1}(\Lambda, \mu ; P)$, the field $f *$ also belongs to $\mathrm{L}^{1}(\Lambda, \mu ; \mathrm{P})$; in fact, $\|\mathrm{f} *\|_{1}=\left\|_{\mathrm{f}}\right\|_{1}$. Hence, we obtain a mapping $*$ (involution) from $L^{1}(\Lambda, \mu ; P)$ into itself.

PROPOSITION 9.8. Under involution $L^{1}(\Lambda, \mu ; P)$ is a Banach *-algebra which we denote by $L^{1}(\Lambda, \mu ; P, I)$.

LEMMA 9.9. If the conditions of 1.16 hold, then $L^{1}(\Lambda, \mu ; P, I)$ is separable. PROOF. The underlying Banach space $L^{1}(\Lambda, \mu)$ is separable by the Corollary to Proposition 2.2 of $[1, \mathrm{p} .20]$.

Next in this section, we turn to a study of identities in $L^{1}(\Lambda, \mu ; P, I)$.

Suppose $A$ has a strong approximate identity. In the proof of Prop. 8.2 of [1], Fel1 shows how to construct an approximate identity for $L^{1}(\Lambda, \mu ; P, I)$. Also observe that this proof does not use the $\Lambda$-continuity of $P$ or $I$. Consequently, we have:

LEMMA 9.10. If $A$ has a strong approximate identity (recall 7.5), then $L^{1}(\Lambda, \mu ; P, I)$ contains an approximate identity (with the same bound).

PROPOSITION 9.11. The Banach *-algebra $\mathrm{L}^{1}(\Lambda, \mu ; \mathrm{P}, \mathrm{I})$ contains an identity if and only if $G$ is discrete and $A$ has an identity.

PROOF. The proof of the corresponding result for classical group algebras 
$\left[12,31^{\circ}\right]$ can be adapted to the vector field context. In particular, the identity $u$ of $A$ and the identity $f$ of $L^{1}(\Lambda, \mu: P, I)$ are related by $f(e)=u$.

For the remainder of this section, we investigate the correspondence between two generalized group algebras whose underlying Fell bundles are connected by a "Fell bundle morphism," i.e. a Banach bundle morphism having the appropriate additional algebraic properties.

Suppose $(B, \tau: Q, J)$ is another Fell bundle over $G$ and $L^{1}(\Lambda, \mu: Q, J)$ is the corresponding generalized group algebra. Let $\phi:(A, \pi) \rightarrow(B, \tau)$ be a Banach bundle morphism as in section 3 (with $\psi=E_{G}$ ). Then we have a field

$$
\Phi=\left\{\Phi_{x}: x \varepsilon G\right\} \quad \varepsilon \Pi_{G} \operatorname{Hom}\left(A_{x}, B_{x}\right),
$$

where $\Phi_{x}=\phi \mid A_{x}, x \in G$. If $f$ is a vector field in $\Pi_{x}$, then it follows that Фf is a vector field in $\Pi_{x}$. Furthermore, the correspondence $f \rightarrow \Phi f$ is linear between the underlying linear spaces of vector fields; notationally, we have

$$
\Phi: \quad \Pi_{\mathbf{x}} \rightarrow \Pi B_{\mathbf{x}}
$$

Now suppose that $\Phi$ is measurable (3.10). Then by 3.14 , we have a linear mapping

$$
\Phi: M(\Lambda, \mu) M(\Delta, \mu)
$$

which is constant on null equivalence classes. If $\Phi$ is continuous, then (3.9)

In particular,

$$
\Phi: C(\Lambda) \rightarrow C(\Delta)
$$

$$
\Phi: C_{c}(\Lambda) \rightarrow C_{c}(\Delta) \text {, }
$$

since $\operatorname{supp}(\Phi f) \subseteq \operatorname{supp}(f)$.

For convenience, let $\|\Phi\|_{G}$ denote

$$
\sup \left\{\left\|\Phi_{\mathbf{x}}\right\|: \mathbf{x} \varepsilon G\right\}
$$

We will say that $\Phi$ is bounded if $\|\Phi\|_{G}<\infty$. Then we have:

PROPOSITION 9.12. If $\Phi(i . e . \phi)$ is measurable and $\phi$ is bounded, then (modulo nullity) $\Phi$ is a bounded, linear mapping of $L^{1}(\Lambda, \mu)$ into $L^{1}(\Delta, \mu)$ with norm at most $\|\Phi\|_{G}$. In particular, if each $\Phi_{x}, x \in G$, is an isometry, then $\Phi$ is an isometry. 
In order that $\Phi$ be a *-algebra homomorphism as we11, we will have to require more of $\Phi$.

DEFINITION 9.13. The mapping

$$
\Phi:(A, \pi ; P, I)(B, \tau ; Q, J)
$$

is a Fell bundle morphism if $\phi:(A, \pi) \rightarrow(B, \tau)$ is a measurable Banach bundle morphism and :

(i) $\phi \mathrm{P}_{\mathrm{x}, \mathrm{y}}=\mathrm{Q}_{\mathrm{x}, \mathrm{y}}(\phi \times \phi)$, i.e. $\Phi_{\mathrm{xy}} \mathrm{P}_{\mathrm{x}, \mathrm{y}}=\mathrm{Q}_{\mathrm{x}, \mathrm{y}}\left(\Phi_{\mathrm{x}} \times \Phi_{\mathrm{y}}\right)$,

(ii) $\phi \mathrm{I}_{\mathbf{x}}=\mathrm{J}_{\mathbf{x}} \phi$, i.e. $\Phi_{\mathbf{x}} \mathrm{I}_{\mathbf{x}}=\mathrm{J}_{\mathbf{x}} \Phi_{\mathbf{x}}$,

for $x, y$ in $G$.

THEOREM 9.14. If $\phi($ as in 9.13) is a bounded Fell bundle morphism, then

$$
\Phi: L^{1}(\Lambda, \mu ; P, I) \rightarrow L^{1}(\Delta, \mu ; Q, J)
$$

is a Banach *-algebra homorphism.

Conversely, suppose $\phi^{-1}: B \rightarrow A$ exists and is a Banach bundle morphism, so that 9.12 and 9.13 apply.

PROPOSITION 9.15. If $\phi, \phi^{-1}$ are bounded Fell bundle morphisms for $(A, \pi ; P, I)$ and $(B, \tau ; Q, J)$ as above, then $L^{1}(\Lambda, \mu ; P, I)$ and $L^{1}(\Delta, \mu ; Q, J)$ are isomorphic Banach *-algebras which are equivalent as Banach spaces. In particular, if each $\Phi$ is an invertible isometry, $x \in G$, then these algebras are isometrically isomorphic.

10. HOMOGENEITY.

Our objective here is to extend the main ideas and results of sections 6 and 9 of [1] to the setting of (measurable) Fell bundles. This will be useful in Section 11 for comparing Leptin bundles with Fell bundles.

Recall that the unitary multipliers $U(A)$ for the Fell bundle $A$ form a group and a topological space with the relativized strong topology.

DEFINITION 10.1. The Fell bundle $A$ is (measurably) homogeneous if:

(i) $A$ has enough unitary multipliers, i.e. $\pi^{\circ}(U(A))=G$.

(ii) The mappings $(m, a) \rightarrow$ ma and $(m, a) \rightarrow$ am of $U(A) \times A$ into $A$ are measurable (as in 3.10). 
REMARK 10.2. For each $x, y$ in $G$ and $m$ in $U_{x}(A)$, the mapping $a \rightarrow$ ma of $A_{y}$ into $A_{x y}$ is a linear isometry. Therefore, if $\pi^{\circ}$ is onto (in particular, if $A$ is homogeneous), then the fibers $\left\{A_{x}: x \in G\right\}$ are all isometrically isomorphic.

LEMMA 10.3. If $A$ has a strong approximate identity and $P$ is ultra-measurable, then $A$ is homogeneous if and only if $A$ has enough unitary multipliers. (Compare with Remark 3 of $[1, p .49]$. )

PROOF. It follows from 8.5 that (ii) of 10.1 is automatically satisfied under the given hypotheses.

REMARK 10.4. In particular, if $A$ is a continuous Fell bundle with approximate identity, then $A$ is homogeneous if and only if $\pi^{\circ} / U(A)$ is onto (7.5). Thus, for the case of such bundles, homogeneity is simply the existence of sufficiently many unitary multipliers. Consequently, this latter property is really the crux of the homogeneity property--both technically and intuitively.

In Section 9 of [1], Fell shows that all continuously homogeneous Fell bundles can be constructed (up to isomorphism) from a given set of "ingredients." We w111 next extend this construction to the measurable case. Moreover, we will do this in the setting of vector fields, describing the underlying continuity structure specifically.

of even greater significance--especially for the needs of Section 13--is the description of the underlying field of Banach spaces. This field is constructed in the same way as is the field in Section 4 of [13]. However, the contexts in which these constructions take place are different in point of view. Consequently, we will also adopt the viewpoint of Section 14 of [13]--namely, that of group representations. This will allow the results of section 13 to follow immediately from [13] and this section.

Let $\mathrm{A}$ be a Banach *-algebra with (bounded) approximate identity. Let $\mathrm{N}$ be a subgroup of the topological group $U(A)$ of unitary multipliers on A Suppose also that $H$ is a topological group extension of $N$ with $q: H \rightarrow H / N$ the canonical epimorphism on the space of right cosets. Assume also that $\mathrm{G}=\mathrm{H} / \mathrm{N}$ is 1 locally 
compact in the usual quotient topology.

REMARK 10.5. The local compactness of $G$ is not assumed by Fell in [1]. This is essential for us here, since $G$ will play the role of a base space $X$, i.e. all of Section 1 must apply to $G$. However, this is not a severe additional assumption, since Fell assumes $G$ is locally compact for the main purposes of [1]--for example, cross-sectional algebras, induced representations, etc.

Given such A, N and H, Fell constructs a field of Banach spaces over $G$ by defining an equivalence relation $\mathrm{N}$ in the space $\mathrm{H} \times \mathrm{A}$. Consistent with our stated point of view, observe that the topological group $\mathrm{N}$ is represented on $\mathrm{A}$ by:

$$
R_{t}(a)=a t^{-1}, a \varepsilon A, t \varepsilon N
$$

Such $R$ is a bounded, strongly continuous representation of $N$ on $A$ which we call the right regular representation. If $(x, a)$ and $(y, b)$ are elements of $\mathrm{H}^{\times} \mathrm{A}$, define:

$$
(\mathrm{x}, \mathrm{a}) \sim(\mathrm{y}, \mathrm{b})
$$

if there exists $t$ in $\mathrm{N}$ for which

$$
b=R_{t}(a)=a t^{-1}, y=t x
$$

This defines an equivalence relation $\approx$ in $H \times A$. Denote the space $(H \times A) / \sim$ of equivalence classes $(x, a)^{\sim}$ by $A$, equipped with the quotient topology from $H \times A$. Observe that $A$ can be viewed as the orbit space of $H \times A$ under the right topological transformation group

$$
(\mathrm{H} \times \mathrm{A}) \times \mathrm{N} \rightarrow \mathrm{H} \times \mathrm{A},
$$

where

$$
((x, a), t) \rightarrow\left(t x, R_{t}(a)\right), a \varepsilon A, t \varepsilon N, x \varepsilon H .
$$

The projection mapping $\pi: A \rightarrow G$ is then given by

$$
\left.\pi(x, a)^{\sim}\right)=q(x), a \varepsilon A, x \varepsilon H .
$$

For each $\xi$ in $G$, let $A_{\xi}=\pi^{-1}(\xi)$. Then $A$ is the disjoint union of the non-empty) fibers $A_{\xi}, \xi \varepsilon G$. If we define

$$
J_{x}: A \rightarrow A_{\xi}, \quad x \in \xi \in G,
$$


by

$$
J_{x}(a)=(x, a)^{\sim}, a \varepsilon A,
$$

then $J_{x}$ is a (well-defined) bijection, since

$$
J_{t x}(a)=J_{x}\left(R_{t}(a)\right), t \varepsilon N, a \varepsilon A, x \varepsilon H .
$$

Therefore, we may transfer the Banach space structure of $A$ fo ${ }^{A_{\xi}}$ by such $J_{x}$. Specifically,

$$
\begin{aligned}
& (x, a)^{\sim}+(x, b)^{\sim}=(x, a+b)^{\sim}, \\
& \beta \cdot(x, a)^{\sim}=(x, \beta a)^{\sim}, \\
& \left\|(x, a)^{\sim}\right\|=\|a\|, \beta \varepsilon \mathbb{C}, a, b \varepsilon A, x \in H .
\end{aligned}
$$

In this way, we obtain a field $\left\{A_{\xi}: \xi \varepsilon G\right\}$ of Banach spaces over $G$.

The continuity structure underlying the bundle $(A, \pi)$ over $G$ is $\Lambda=C(\Lambda)=\operatorname{CS}(G, A)$. This is obtained from $C(H, A)$ in the following way. For $D$ a "saturated" subset of $\mathrm{H}$, i.e. $\mathrm{D}$ a union of $\mathrm{N}$-cosets, define

$$
C_{N}(D, A)=\left\{f \varepsilon C(D, A): f(t x)=R_{t}(f(x)), x \in H, t \varepsilon N\right\} \text {. }
$$

Then $C_{N}(D, A)$ is a linear subspace of $C(D, A)$. For $f$ in $C_{N}(D, A)$, define

$$
\mathrm{f}^{\sim}(\mathrm{x})=(\mathrm{x}, \mathrm{f}(\mathrm{x}))^{\sim}=\mathrm{J}_{\mathrm{x}}(\mathrm{f}(\mathrm{x})), \mathrm{x} \in \mathrm{H} \text {. }
$$

The mapping $\mathrm{f}^{\sim}$ is constant on cosets and hence, defines a mapping $\mathrm{f}^{\sim}: G^{\prime} A$ which is easily seen to be a cross-section. Since $\mathrm{f}^{\sim}$ is also the composition of continuous mappings, it is also continuous, i.e. $f \rightarrow f^{\sim}$ is a mapping of $C_{N}(D, A)$ into $\operatorname{CS}(\mathrm{q}(\mathrm{D}), \mathrm{A}) . \quad$ In particular,

$$
\left\{f^{\sim}: f \varepsilon C_{N}(H, A)\right\} \subseteq C(\Lambda)=C S(G, A)
$$

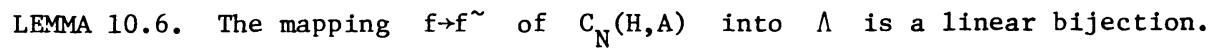
Furthermore, if $h \varepsilon \Lambda$, then $h=f^{\sim}$, for the unique $f$ in $C_{N}(H, A)$ given by

$$
f(x)=J_{x^{-1}}(h(q(x))), x \varepsilon H
$$

Next suppose we are given a mapping $\tau$ of $\mathrm{H}$ into the topological group Aut $_{1}$ (A) of isometric *-automorphisms of A having the following "admissibility" properties :

(i) $\tau$ is a group homomorphism.

(ii) $\tau_{t}$ (a) $\left.=\operatorname{tat}^{-1}=\mathrm{R}_{t}\left(\mathrm{R}_{t}\left(\mathrm{a}^{*}\right)\right) *\right)$, $a \varepsilon A, t \varepsilon N$. 
(iii) $\tau_{x}^{\prime}(t)=x t x^{-1}, x \in H, t \varepsilon N$, where $\tau_{x}^{\prime}$ is the unique extension of $\tau_{x}$ to

$M(A)$ defined by:

$$
\tau_{x}^{\prime}(m) \tau_{x}(a)=\tau_{x}(m a), a \varepsilon A, m \varepsilon M(A) .
$$

The *-algebra structure of $A$ is defined by means of $\tau$. In terms of operator fields, this structure is given by:

$$
\begin{aligned}
& P_{\xi, n}: A_{\xi} \times A_{\eta} \rightarrow A_{\xi \eta}, \\
& I_{\xi}: A_{\xi} \rightarrow A_{\xi}-1, \eta, n \varepsilon G .
\end{aligned}
$$

where

$$
\begin{aligned}
& P_{\xi, \eta}\left((x, a)^{\sim},(y, b)^{\sim}\right)=\left(x y, a \cdot \tau_{x}(b)\right)^{\sim}, \\
& I_{\xi}\left((x, a)^{\sim}\right)=\left(x^{-1}, \tau^{-1}(a) *\right), \cdot a, b \varepsilon A, x \varepsilon \xi, y \varepsilon n:
\end{aligned}
$$

LEMMA 10.7. The fields $P$ and $I$ are product and involution fields respectively.

EXAMPLE 10.8. (One-dimensional fibers $[1, p .75]$ ) If $A$ is the complex numbers $\mathbb{C}$, then $N=U(A)$ is the circle group. Also, $\tau$ must be trivial, since Aut ${ }_{1}(\mathbb{C})$ is trivial. Thus, for $H$ as above, we have:

$$
\begin{aligned}
& C_{N}(H, A)=\{f \in C(H): f(t x)=\bar{t} f(x), t \varepsilon N, x \in H\}, \\
& P_{\xi, \eta}\left((x, a)^{\sim},(y, b)^{\sim}\right)=(x y, a b)^{\sim}, \\
& I_{\xi}\left((x, a)^{\sim}\right)=\left(x^{-1}, \bar{a}\right)^{\sim}, x \in \xi \varepsilon G, y \varepsilon \eta \varepsilon G, a, b \in \mathbb{C} .
\end{aligned}
$$

In order to motivate suitable definitions of measurability for $\tau$ (as above), consider the following characterizations of continuity:

PROPOSITION 10.9. Let $\tau: H \rightarrow \mathrm{Aut}_{1}(\mathrm{~A})$. The following are equivalent for $\mathrm{x}_{\mathrm{o}}$ in $\mathrm{H}$ :

(i) $\tau$ is strongly continuous at $x_{0}$.

(ii) The mapping of $H \times A$ into $A$ given by $(x, a) \rightarrow \tau_{x}(a)$ is continuous on $\left\{x_{0}\right\} \times A$.

(iii) For each $f$ in $\mathrm{C}(\mathrm{H}, \mathrm{A})$, the mapping of $\mathrm{H} \times \mathrm{H}$ into $\mathrm{A}$ given by $(x, y) \rightarrow \tau_{x}(f(y))$ is continuous on $\left\{x_{0}\right\} \times H$. 
(iv) For each $a$ in $A$, the mapping of $H$ into $A$ given by $x \rightarrow(x, \tau(a))^{\sim}$ is continuous at $x_{0}$.

(v) The mapping of $H \times A$ into $A$ given by $(x, a) \rightarrow\left(x, \tau_{x}(a)\right)^{\sim}$ is continuous on $\left\{\mathrm{x}_{0}\right\} \times \mathrm{A}$.

Moreover, under the above conditions, for each $\mathrm{f}, \mathrm{g}$ in $\mathrm{C}(\mathrm{H}, \mathrm{A})$, the mapping of $\mathrm{H} \times \mathrm{H}$ into $\mathrm{A}$ given by $(\mathrm{x}, \mathrm{y}) \rightarrow \mathrm{f}(\mathrm{x}) \cdot \tau_{\mathrm{x}} \mathrm{g}(\mathrm{y})$ is continuous on $\left\{\mathrm{x}_{\mathrm{o}}\right\} \times \mathrm{H}$.

PROOF. All implications are straightforward with the exception of "(iv) implies (i)", which follows from Lemma 9.1 of $[1, p .70]$.

In view of 10.9 , we propose the following:

DEFINITION 10.10. A mapping $\tau: \mathrm{H} \rightarrow$ Aut $_{1}(\mathrm{~A})$ is measurable if, for each a in A , the mapping $x \rightarrow \tau_{x}(a)$ is measurable in the following sense: For each compact subset $K$ of $G$ and $\varepsilon>0$, there exists a compact subset $K_{\varepsilon, a}$ of $K$ such that $\mu\left(\mathrm{K}-\mathrm{K}_{\varepsilon, \mathrm{a}}\right)<\varepsilon$ and the mapping $\mathrm{x} \rightarrow \tau_{\mathrm{x}}(\mathrm{a})$ is continuous on $\mathrm{q}^{-1}\left(\mathrm{~K}_{\varepsilon, \mathrm{a}}\right)$.

There is also a stronger notion of measurability as in the case of bundle morphisms.

DEFINITION 10.11. A mapping $\tau: H \rightarrow$ Aut $_{1}(\mathrm{~A})$ is ultra-measurable if, for each compact subset $K$ of $G$ and $\varepsilon>0$, there exists a compact subset $K_{\varepsilon}$ of $K$ such that $\mu\left(\mathrm{K}-\mathrm{K}_{\varepsilon}\right)<\varepsilon$ and $\tau$ is continuous on $\mathrm{q}^{-1}\left(\mathrm{~K}_{\varepsilon}\right)$ to Aut ${ }_{1}(\mathrm{~A})$ for the topology of pointwise convergence, i.e.

$$
\tau: q^{-1}\left(\mathrm{~K}_{\varepsilon}\right) \rightarrow \operatorname{Aut}_{1}(\mathrm{~A})
$$

is strongly continuous.

As before, these two notions of measurability are equivalent in the presence of separability.

LEMMA 10.12. If $\tau$ is measurable and $A$ is separable, then $\tau$ is ultrameasurable.

PROOF. This follows from Prop. 2 of $[14, \mathrm{p} .170]$ as in the proof of 3.13 . PROPOSITION 10.13. Suppose $\tau$ is an admissible mapping of $H$ into Aut ${ }_{1}(A)$. Let $\mathrm{P}$ and $\mathrm{I}$ be as above. 
(1) If $\tau$ is measurable, then the onerator fields $P$ and $I$ are measurable.

(2) If $\tau$ is ultra-measurable, then $P$ and $I$ are ultra-measurable.

(3) If $\tau$ is continuous, then $P$ and $I$ are continuous (relative to $\Lambda$ ).

It follows from the above that the ingredients $(A, N, H, \tau$ ) yield a Fell bundle

$A$ when $\tau$ is admissible and measurable. The obvious next question is whether or not $A$ is homogeneous. For each $y$ in $H$, define:

$$
M_{y}(x, a)^{\sim}=\left(y x, \tau_{y}(a)\right)^{\sim}
$$

and

$$
(x, a) \sim_{y}=(x y, a)^{\sim}, x \in H, a \varepsilon A
$$

LEMMA 10.14. For each $y$ in $H, M_{y}$ is a unitary multiplier of $A$, i.e. $M_{y} \varepsilon U(A)$

PROOF. See p. 71 of [1].

The previous lemma shows that $\pi^{\circ}(U(A))=G$ for the bundle $A$. Consequently, A will be measurably homogeneous if (ii) of 10.1 holds. In particular, this will be the case if (1) $\tau$ is ultra-measurable, and (2) A has a strong approximate identity (10.3). (Note that if $\left\{u_{j}\right\}$ is an approximate identity in $A$, then $\left\{\left(e, u_{j}\right)^{\sim}\right\}$ is an approximate identity in $\left.A_{.}\right)$These appear to be false in general-unless $\tau$ is continuous (7.5). Hence, it seems unlikely that $A$ is homogeneous in the techncial sense of 10.1. However, in view of 10.4 , we feel that $A$ is as homogeneous as it can be under the circumstances.

In the opposite direction, Fell shows [1, Thm.9.1] that every (continuously) homogeneous Banach *-algebraic bundle is isomorphic to one obtained from ingredients $(\mathrm{A}, \mathrm{N}, \mathrm{H}, \tau)$ with $\tau$ continuous. In particular, the group extension $\mathrm{H}$ is chosen to be $U(A)$. This is not possible in the measurable setting, since it is not clear that $U(A)$ is a topological group. Perhaps there is another way of obtaining a measurable generalization of his Theorem 9.1. For example, it may be possible to replace $U(A)$ by its image in $U\left(L^{1}(\Lambda, \mu: P, I)\right)[2, p p .137-139]$. We refer the reader to Section $V$ of [5] for further information regarding this possibility. 


\section{UNITARY FACTOR SYSTEMS.}

The other two (equivalent) bundle constructions referred to in the introduction are due to Leptin [3] and to Busby and Smith [4]. These constructions are quite similar to each other, but very different from that of Fell. Although their objects are quite familiar (vector-valued functions), their algebraic operations are not. In this section, we review Leptin's approach (as in [5]) and show how it gives rise to a homogeneous Fell bundle.

Let $G$ be a locally compact group and $A$ a Banach *-algebra with approximate identity. Let $M(A), U(A)$ and $A_{1} t_{1}(A)$ be as above. The ingredients for the Leptin construction [5] are G,A together with the following:

DEFINITION 11.1. A unitary factor system $(T, W)$ for $(G, A)$ is a pair of strongly measurable mappings

$$
T: G \rightarrow A u t_{1}(A), \quad W: G \times G \rightarrow U(A)
$$

satisfying:

$$
\begin{aligned}
& \text { (i) } \left.\left.W_{x y, z} \cdot T_{z}-1 W_{x, y}\right)=W_{x, y z} \cdot W_{y, z}, x, y, z \text { in } G \text { (multiplication in } M(A)\right) \\
& \text { (ii) } a W_{x, y}=W_{x, y} T_{x, y}(a) \text {, where } T_{x, y}=T_{y}-1{ }_{x}-1{ }^{T}{ }_{y}^{-1} x^{-1} \text {, for } x, y \text { in } G \text {, a in } A \\
& \text { (iii) } W_{e, x}=W_{x, e}=\left(E_{A}, E_{A}\right), T_{e}=E_{A}, x, y \text { in } G \text {, a in } A .
\end{aligned}
$$

REMARK 11.2. As in the case of $\tau$, there is a notion of measurability for W, T between strong measurability and strong continuity--namely Bourbaki measurability $[10, \mathrm{p} .169]$. This is what we will call ultra-measurability for $W, T$ for obvious reasons. As in 10.12 , we have:

LEMMA 11.3. For $\mathrm{W}($ resp. $T$ ) strongly measurable and A separable, W(resp. T) is ultra-measurable.

Let $(A, \pi)$ be the trivial bundle GXA over $G$ (product topology) with $\pi(x, a)=x, x \in G, a \in A$. Let $A_{x}$ be the Banach space $\{x\} \times A$ canonically isomorphic to $A, x \in G$. For each $f$ in $C(G, A)$, let $f_{G}$ be the mapping

$$
E_{G} \times f:: \times G \rightarrow G \times A
$$


Then $\left\{\mathrm{f}_{G}: \mathrm{f} \varepsilon \mathrm{C}(\mathrm{G}, \mathrm{A})\right\}$ is a continuity structure $\Lambda$ in $\mathrm{IA}_{\mathrm{x}}$ for which

$$
\Lambda=C(\Lambda)=\operatorname{Cs}(G, A)
$$

The algebraic structure in $A$ is defined as follows in terms of operator fields :

$$
\begin{aligned}
& (x, a)(y, b)=P_{x, y}((x, a),(y, b))=\left(x y, W_{x, y^{T}}-1(a) b\right) \\
& (x, a) *=I_{x}(x, a)=\left(x^{-1}, W^{*}{ }_{x, x^{-1}} T^{-1}\left(a^{*}\right)\right), x, y \in G, a, b \varepsilon A .
\end{aligned}
$$

PROPOSITION 11.4. (1) The field P (resp.I) is a measurable product (resp. involution) field.

(2) If $W, T$ are ultra-measurable, then so are $P, I$.

(3) If $\mathrm{W}, \mathrm{T}$ are (strongly) continuous, then so are $\mathrm{P}, \mathrm{I}$ (relative to $\Lambda$ ).

PROOF. Parts (1) and (3) are straightforward. Part (2) is proved as in Section 10.

The resulting Fell bundle $(A, \pi ; P, I)$ will be called a Leptin bundle and will be denoted by $(G, A: T, W)$.

PROPOSITON 11.5. Every Leptin bundle has enough unitary multipliers.

PROOF. As on P. 329 of [5], for $z$ in $G$, define:

$$
\begin{aligned}
& M_{z}(x, a)=\left(z x, W_{z, x} a\right), \\
& (x, a) M_{z}=\left(x z, W_{x, z} T_{z}-1(a)\right),(x, a) \varepsilon G \times A .
\end{aligned}
$$

Hence, every Leptin bundle is a homogeneous Fell bundle in the weak sense of having enough unitary multipliers.

Observe that if $\left\{u_{j}\right\}$ is an approximate identity in $A$, then $\left\{\left(e, u_{j}\right)\right\}$ is an approximate identity in $A$, where:

$$
\left(e, u_{j}\right)(x, a)=\left(x, T_{x}-1\left(u_{j}\right) a\right)
$$

and

$$
(x, a)\left(e, u_{j}\right)=\left(x, a u_{j}\right),(x, a) \in A \text {. }
$$

PROPOSITION 11.6. If $T$ is strongly continuous, then $\left\{\left(e, u_{j}\right)\right\}$ is a strong 
approximate identity for $A$.

PROOF. This can be proved directly or obtained as a consequence (by 7.5 ) of the following result.

PROPOSITION 11.7. Let $(G, A ; T, W)$ be a Leptin bundle. Then:

(1) $C(\Lambda)$ is locally a right A-module.

(2) If $T$ is strongly continuous, then $C(\Lambda)$ is locally a (two-sided) A-module. COROLLARY 11.8. If $A$ is separable and $T$ is strongly continuous, then the Leptin bundle $(\mathrm{G}, \mathrm{A} ; \mathrm{T}, \mathrm{W})$ is homogeneous in the sense of 10.1 .

PROOF. This follows from 10.3 together with $11.3,11.4,11.5$, and 11.6 .

PROPOSITION 11.9. Every Leptin bundle is saturated.

PROOF. This would normally follow from Prop. 11.5 of [2]. However, since the validity of this proposition is questionable in the case of measurable bundles, its conclusion has to be verified directly.

The generalized $\mathrm{L}^{1}$-algebra $\mathrm{L}^{1}(\mathrm{G}, \mathrm{A} ; \mathrm{T}, \mathrm{W})$ corresponding to the underlying Leptin bundle is the Banach space $L^{1}(G, A, \mu)$ with convolution and involution (for right Haar measure) given by:

$$
\begin{aligned}
& f * g(x)=\int_{G^{T V}}{ }^{-1}, y^{T} y^{-1} f\left(x y^{-1}\right) g(y) d \mu(y), \\
& f *(x)=\delta(x)^{-1} W^{*}{ }_{x}^{-1}, x^{T_{x}^{-1}\left(f\left(x^{-1}\right) *\right), x \in G,}
\end{aligned}
$$

for $f$ and $g$ in $L^{1}(G, A, \mu)$. This Banach *-algebra is isometrically isomorphic to our generalized group algebra $L^{1}(\Lambda, \mu ; P, I)$ of Section 9 by the mapping

$$
\mathrm{f} \rightarrow \mathrm{f}_{G}, \quad \mathrm{f} \varepsilon \mathrm{L}^{1}(G, \mathrm{~A} ; \mathrm{T}, \mathrm{W}) .
$$

Moreover, by 11.6 and 9.11, $\mathrm{L}^{1}(\mathrm{G}, \mathrm{A} ; \mathrm{T}, \mathrm{N})$ will have an approximate identity if $\mathrm{T}$ is continuous.

As observed in Section IV of [5], certain homogeneous bundles give rise to equivalent Leptin bundles. Suppose $(A, N, H, \tau)$ are the ingredients for a homogeneous Banach *-algebra bundle as in Section 10 . Let $(A, \pi ; P, I)$ denote the corresponding Fell bundle, where $A=H \times A / \sim$ and $\pi(x, a)^{2}=q(x), x \varepsilon H, a \varepsilon A$. 
Assume also that $\mathrm{G}=\mathrm{H} / \mathrm{N}$ is locally compact (as before) and that there exists a measurable cross-section $\sigma: G \rightarrow H$. If we define

$$
\mathrm{T}_{\xi}=\tau_{\sigma\left(\xi^{-1}\right)}
$$

and

$$
w_{\xi, \eta}=\tau_{\sigma(\xi \eta)^{-1} \sigma(\xi) \sigma(\eta)}, \quad \xi, n \varepsilon G,
$$

then we obtain a Leptin bundle $(G, A ; T, W)$ [5,P.333]. Leinert also shows that the group algebras $L^{1}(A, \pi ; P, I)=L^{1}(\Lambda, \mu ; P, I)$ and $L^{1}(G, A ; T, W)$ are isometrically isomorphic. This can also be concluded from the following.

Define

$$
\phi: \mathrm{G} \times \mathrm{A} \rightarrow \mathrm{A}
$$

by

$$
\phi(\xi, a)=(\sigma(\xi), a)^{\sim},(\xi, a) \varepsilon G \times A
$$

Then it is easy to verify that $\phi$ is an invertible, bi-measurable Fell bundle isomorphism. Hence, the algebra isomorphism follows from 9.15 .

\section{TWISTING PAIRS.}

Let $G$ and $A$ be as in Section 11. The Busby-Smith approach [4] to constructing a generalized group algebra from $L^{1}(G, A, \mu)$ requires the following:

DEFINITION 12.1. A twisting pair $(\mathrm{S}, \mathrm{V})$ for $(\mathrm{G}, \mathrm{A})$ is a pair of strongly measurable mappings

$$
\mathrm{S}: \mathrm{G} \rightarrow \operatorname{Aut}_{1}(\mathrm{~A}), \mathrm{V}: \mathrm{G} \times \mathrm{G} \rightarrow \mathrm{U}(\mathrm{A})
$$

satisfying:

(i) $\mathrm{S}_{\mathrm{x}}\left(\mathrm{v}_{\mathrm{y}, \mathrm{z}}\right) \mathrm{v}_{\mathrm{x}, \mathrm{yz}}=\mathrm{v}_{\mathrm{x}, \mathrm{y}} \mathrm{V}_{\mathrm{xy}, \mathrm{z}}$,

(multiplication in $\mathrm{M}(\mathrm{A})$ )

(ii) $\left(\mathrm{S}_{\mathrm{x}} \mathrm{S}_{\mathrm{y}}(\mathrm{a})\right) \mathrm{v}_{\mathrm{x}, \mathrm{y}}=\mathrm{v}_{\mathrm{x}, \mathrm{y}} \mathrm{S}_{\mathrm{xy}}(\mathrm{a})$,

(iii) $v_{x, e}=v_{e, x}=\left(E_{A}, E_{A}\right), S_{e}=s_{A}$, for $x, y, z \varepsilon G, a \varepsilon A$.

LEMMA 12.2. For V(resp. S) strongly measurable and A separable, V(resp.S) is ultra-measurable, i.e. Bourbaki measurable (recall 11.2). 
As in Section 11 , the underlying bundle $(A, \pi)$ is the product bundle $(G \times A, \pi)$ with continuity structure

$$
\Lambda=\left\{f_{G}: f \in C(G, A)\right\} .
$$

The algebraic structure in $A$ is defined by operator fields $P$ and $I$ as follows:

$$
\begin{gathered}
(x, a) \cdot(y, b)=P_{x, y}((x, a),(y, b))=\left(x y, a S_{x}(b) v_{x, y}\right), \\
(x, a) *=I_{x}((x, a))=\left(x^{-1}, v^{*} x^{-1}, x_{x} S^{-1}\left(a^{*}\right)\right),
\end{gathered}
$$

for $x, y$ in $G$ and $a$ in $A$.

Twisting pairs $(S, V)$ for $(G, A)$ are in one-to-one correspondence with unitary factor systems $(T, W)$ for $(G, A)$ [5,pp.317-318]. This correspondence is given by

$$
\begin{aligned}
& T_{x}=s_{x}^{-1} \\
& w_{x, y}=s_{x y}^{-1} v_{x, y}, x, y \in G .
\end{aligned}
$$

Thus, $S$ and $V$ are strongly continuous (resp. measurable) if and only if $T, W$ are. Moreover:

LEMMA 12.3. The mappings $\mathrm{S}$ and $\mathrm{V}$ are ultra-measurable if and only if the mappings $\mathrm{T}$ and $\mathrm{W}$ are.

PROPOSITION 12.4. (1) The field P(resp.I) is a measurable product (resp. involution) field.

(2) If $\mathrm{S}$ and $\mathrm{V}$ are ultra-measurable, then so are $\mathrm{P}$ and $\mathrm{I}$.

(3) If $S$ and $V$ are strongly continuous, then so are $P$ and $I$ (relative to $\Lambda$ ).

The resulting Fell bundle $(A, \pi ; P, I)$ will be called a Busby-Smith bundle and will be denoted by $(G, A ; S, V)$.

THEOREM 12.5. For corresponding pairs $(\mathrm{T}, \mathrm{W})$ and $(\mathrm{S}, \mathrm{V})$, the Leptin bundle $(G, A ; T, W)$ is measurably isomorphic to the Busby-Smith bundle $(G, A ; S, V)$.

PROPOSITION 12.6. The following are equivalent for $x_{0}$ in $G$ :

(1) $\mathrm{T}$ is strongly continuous at $\mathrm{x}_{\mathrm{o}}$.

(2) S is strongly continuous at $x_{0}$. 
(3) $\Phi$, i.e. $\phi$ is continuous at $x_{0}$.

PROOF. (1) is equivalent to (2) since $\mathrm{S}_{\mathrm{x}}^{-1}=\mathrm{T}_{\mathrm{x}}^{-1}, \mathrm{x} \varepsilon \mathrm{G} \cdot$ (3) is equivalent to (2) since the mapping $x \rightarrow S_{x}(h(x))$ is continuous at $x_{0}$, for $h$ in $C(G, A)$, if and only if $\Phi_{h}$ is continuous at $x_{0}$.

COROLLARY 12.7. The Fell bundles (G,A;T,W) and (G,A;S,V) are homomorphically isomorphic if $\mathrm{T}$ (equivalently $\mathrm{S}$ ) is strongly continuous. (Note that the bundles don't have to be continuous Fell bundles in this case.)

REMARK 12.8. Clearly, 11.5 through 11.9 are valid also for Busby-Smith bundles. The twisted group algebra $\mathrm{L}^{1}(\mathrm{G}, \mathrm{A} ; \mathrm{S}, \mathrm{V})$ corresponding to the underlying BusbySmith bundle is the Banach space $L^{1}(G, A, \mu)$ with product and involution (for right Haar measure) given by

$$
f \cdot g(x)=\int_{G} f\left(x y^{-1}\right) S_{x y}-1(g(y)) V\left(x y^{-1}, y\right) d \mu(y)
$$

and

$$
f *(x)=\delta(x)^{-1} V\left(x, x^{-1}\right) * S_{x}\left(f\left(x^{-1}\right)\right) *, \quad x \in G
$$

for $f$ and $g$ in $L^{1}(G, A, \mu)$. The algebra $L^{1}(G, A ; S, V)$ is isomorphic to the algebra $L^{1}(G, A ; T, W) \quad(9.13)$ by the mapping

$$
f \rightarrow\left[x \rightarrow S^{-1}(f(x))\right], \quad f \in L^{1}(G, A ; S, V)
$$

and hence, to our algebra $L^{1}(\Lambda, \mu ; P, I)$ by the same mapping. Moreover, by 9.11, 11.6 , and 12.5, $\mathrm{L}^{1}(\mathrm{G}, \mathrm{A} ; \mathrm{S}, \mathrm{V})$ will have an approximate identity if $\mathrm{S}$ is strongly continuous.

EXAMPLE $12.9[5, \mathrm{P} \cdot 330]$. Let $\mathrm{A}$ be $\mathrm{L}^{1}(\mathbb{Z})$ and $\mathrm{G}$ the circle group identified with $[0,1]$ under addition modulo 1 . Define $S_{x}=T_{x}=E_{A}, x \in G$, and $\mathrm{V}, \mathrm{W}: \mathrm{G} \times \mathrm{G} \rightarrow \mathrm{U}(\mathrm{A})$ by

$$
\begin{aligned}
& \mathrm{v}_{\mathrm{x}, \mathrm{y}^{\mathrm{a}}}=\mathrm{w}_{\mathrm{x}, \mathrm{y}^{\mathrm{a}}}=\mathrm{a}, \\
& \mathrm{v}_{\mathrm{x}, \mathrm{y}^{\mathrm{a}}}=\mathrm{w}_{\mathrm{x}, \mathrm{y}} \mathrm{a}^{\mathrm{a}}=\mathrm{a}_{1}, \mathrm{x}+\mathrm{y} \geq 1,
\end{aligned}
$$

where

$$
a_{1}(n)=1+n, \quad n \in \mathbb{Z}
$$


Then $v_{x, y} a=a V_{x, y}, a \varepsilon A$, and $(S, V)$ is a twisting pair (equivalently, $(T, W)$ is a unitary factor system) for $(G, A)$. The mapping $V$ is not strongly continuous at any point $(x, y)$ in $G \times G$ where $x+y=1$. However, $V$ is ultra-measurable (11.2). In the next section (13.2), we will see that $L^{1}(G, A ; S, V)$ is isometrically isomorphic to the group algebra $L^{1}(\mathbb{R})$.

13. GROUP EXTENSIONS.

Suppose $\mathrm{N}$ is a closed normal subgroup of the locally compact group $\mathrm{H}$ with $\mathrm{G}=\mathrm{H} / \mathrm{N}$ (right cosets) and $\mathrm{q}: \mathrm{H} \rightarrow \mathrm{G}$ the quotient mapping. Let $\rho, \nu$ be right Haar measures for $\mathrm{N}, \mathrm{H}$ respectively. Let $\mu$ be the right Haar measure on $G$ defined [15] by

$$
\int_{H} f(x) d \nu(x)=\int_{G} \int_{N} f(t x) d \rho(t) d \mu(q(t)),
$$

for $f$ in $C_{C}(G)$. Let $\delta_{H}, \delta_{G}, \delta_{N}$ be the respective modular functions for $\mathrm{H}, \mathrm{G}, \mathrm{N}$. Of course, $\delta_{\mathrm{H}} / \mathrm{N}=\delta_{\mathrm{N}}$.

On $\mathrm{p} .77$ of $[1]$, Fell shows how to construct ingredients $(\mathrm{A}, \mathrm{N}, \mathrm{H}, \tau)$ for a homogeneous Banach *-algebraic bundle $(A, \pi ; \cdot, *)$ called the $(H, N)$-group extension bundle. Specifically, $A=L^{1}(N, \rho), N$ is identified with a subgroup of $U(A)$ and

$$
\tau_{x}(a)(t)=\delta(x) a\left(x^{-1} t x\right),
$$

where

$$
\delta(x)=d \rho\left(x_{t x}^{-1}\right) / d \rho(t), \quad x \varepsilon H, t \varepsilon N, a \varepsilon A
$$

Fell then verifies directly that the cross-section algebra $L^{1}(A, \mu ; \cdot, *)$ is isometrically isomorphic to the group algebra $\mathrm{L}^{1}(\mathrm{H}, \nu)$.

In this section, we will accomplish these two tasks in very different ways. We will show that the mapping $\tau$ arises naturally from a certain induction of Banach space representations $([16, \S 3]$ and $[13, \S 4])$. As a consequence of this approach, it will then be automatic that the previous $L^{1}$-spaces are isometrically isomorphic. Transferring the *-algebra structure from $L^{1}(H, \nu)$ to $L^{1}(A, \mu ; \cdot, *$ ) (i.e. to $\left.L^{1}(\Lambda, \mu ; P, I)\right)$ then completes the picture.

Let $G, H, N$ be as above with $E=\{e\}$ and $1_{E}(e)=1$. Then $1_{E}$ is both the identity representation of $E$ and the regular representation of $E$ on $L^{1}(E)$. The 
$\mathrm{L}^{1}$-induction [16] of $1_{E}$ from $\mathrm{E}$ up to $\mathrm{N}$ is then the (right) regular representation $\mathrm{R}_{\mathrm{N}}$ of $\mathrm{N}$ on $\mathrm{L}^{1}(\mathrm{~N}, \rho)[16,4.5]$. Consequently, $\mathrm{L}^{1}$-induction of $\mathrm{R}_{\mathrm{N}}$ from $N$ up to $H$ is the regular representation $R_{H}$ of $H$ on $L^{1}(H, v)[16,6,4]$. The representation space $F^{1}\left(H, R_{N}\right)$ of $R_{H}$ (by this induction) is given as in Section 3 of [16]. On the other hand, the $L^{1}$-induction of $1_{E}$ from $E$ directly up to $H$ is also (modulo equivalence) the representation $R_{H}[16,4.5]$. Therefore, the representation spaces $L^{1}(H, \nu)$ and $F^{1}\left(H, R_{N}\right)$ must be isometrically isomorphic Banach spaces. If

$$
T: L^{1}(H, \nu) \rightarrow F^{1}\left(H, R_{N}\right)
$$

denotes the isomorphism, then it is given [16,p.72] by

$$
(T f)(x)(t)=f(t x), \quad x \in H, t \in N, f \in L^{1}(H, \nu) .
$$

In particular, if $f \in C_{C}(H)$, then so is $T f$ and the images of such $f$ by $T$ are dense in $\mathrm{F}^{1}\left(\mathrm{H}, \mathrm{R}_{\mathrm{N}}\right)$.

Since $L^{1}(H, \nu)$ has a *-algebra structure, it may be transferred to $F^{1}\left(H, R_{N}\right)$ via $\mathrm{T}$ by requiring:

$$
\begin{aligned}
(T f) \cdot(T g) & =T(f \cdot g), \\
(T f) * & =T(f *), \quad f, g \varepsilon L^{1}(H, \nu) .
\end{aligned}
$$

In the case of involution, for $f$ in $\mathrm{C}_{c}(\mathrm{H})$, we have:

$$
(\mathrm{Tf}) *(x)=\delta_{H}(x)^{-1}\left[\mathrm{Tf}\left(\mathrm{x}^{-1}\right)\right] *\left(\mathrm{x}^{-1}(\cdot) \mathrm{x}\right), \quad \mathrm{x} \varepsilon \mathrm{H} .
$$

If, for $y$ in $H$ and $a$ in $L^{1}(N, \rho)$, we define

$$
\tau_{y} a(s)=\delta\left(y^{-1}\right) a\left(y^{-1} s y\right), \quad s \in N,
$$

then, combining the above, we obtain [15]

$$
(T f) *(x)=\delta_{G}\left(q(x)^{-1}\right) \tau_{x}\left(\operatorname{Tf}\left(x^{-1}\right) *\right), \quad x \in H .
$$

In the case of multiplication, for $f, g$ in $C_{c}(H)$, we have:

$$
(T f) \cdot(T g)(x)=\int_{G} \operatorname{Tf}\left(y^{-1}\right){ }^{*}{ }_{N}{ }^{\top}{ }^{-1}(\operatorname{Tg}(y x)) d \mu(q(y)),
$$

where ${ }^{*}$ denotes the usual convolution in $A=L^{1}(N, \rho)$.

Next observe that the representation $R_{N}$ of $N$ on $A$, which is given by

$$
R_{t}(a)=a(\cdot t), \quad a \varepsilon L^{1}(N, \rho), \quad t \in N,
$$


is also given by

$$
R_{t}(a)=a \cdot t^{-1}
$$

where the right side is the action of the multiplier in $U\left(L^{l}(N, \rho)\right)$ corresponding to $t^{-1}$ on the function $a$. Hence, by the beginning of Section 10 (or Section 4 of [13]), there exist:

(1) a field $\left\{A_{\xi}: \xi \varepsilon_{G}\right\}$ of Banach spaces over $G$, each isomorphic to $A=L^{1}(N, \rho)$.

(2) a continuity structure $\Lambda$ in $\Pi_{G}{ }^{A} \xi$ as in 10.6. Moreover, the disjoint union of the $A_{\xi}$ with the $\Lambda$-topology is precisely $A=H \times A / \sim$ (as in Section 10) with the quotient topology.

PROPOSITION 13.1. The mapping $\tau$ defined above is a strongly continuous homomorphism of $\mathrm{H}$ into $\mathrm{Aut}_{1}$ (A) satisfying:

(i) $\tau_{t}(\mathrm{a})=\mathrm{tat}^{-1}, \mathrm{t} \varepsilon \mathrm{N}, \mathrm{a} \varepsilon \mathrm{A}$.

(ii) $\tau_{x}^{\prime}(t)=x t x^{-1}, x \in H, t \varepsilon N$.

PROOF. Left to the reader. Compare with example 3 on p. 77 of [1]. As a consequence of the above, $(\mathrm{H}, \mathrm{N}, \mathrm{A}, \tau)$ is the set of ingredients of a (continuously) homogeneous Banach *-algebraic bundle $(A, \pi ; \bullet, *)$. The underlying product and involution fields $P$ and $I$ are given as in Section 10 . We thus obtain the generalized group algebra $L^{1}(\Lambda, \mu ; P, I)$. Since the constructions of $\left\{A_{\xi}: \xi \varepsilon_{G}\right\}$ and $\Lambda$ above are just special cases of those given in Section 4 of [13], it follows that $F^{1}\left(H, R_{N}\right)$ is isometrically isomorphic to $L^{1}(\Lambda, \mu)$ as Banach spaces. If $S$ is the isomorphism, then $[13,4.12]$

$$
\operatorname{Sf}(\xi)=(x, f(x))^{\sim}, \quad x \varepsilon \xi \varepsilon G,
$$

for $f$ in $F^{1}\left(H, R_{N}\right)$ continuous with compact support module $N$. Thus, the *-algebra structure of $L^{1}(H, \nu)$ may in turn be transferred to $L^{1}(\Lambda, \mu)$ by $\mathrm{V}=\mathrm{ST}$. In particular, for $\mathrm{f}, \mathrm{g}$ in $\mathrm{C}_{\mathrm{c}}(\mathrm{H})$, we obtain:

$$
(\mathrm{Vf}) *(\xi)=V(f *)(\xi)=S(T(f *))(\xi)=(x, T f *(x))^{\sim}, x \varepsilon \xi,
$$

and

$$
\mathrm{Vf} \cdot \mathrm{Vg}=\mathrm{V}\left(\mathrm{f}{ }_{\mathrm{H}} \mathrm{g}\right)=\mathrm{S}\left(\mathrm{T}\left(\mathrm{f}{ }_{\mathrm{H}} \mathrm{G}\right)\right) \text {. }
$$

We may then verify that the two *-algebra structures on $L^{1}(\Lambda, \mu)$ are 


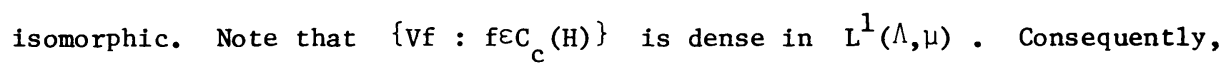
$\mathrm{L}^{1}(\mathrm{H}, \nu)$ and $\mathrm{L}^{1}(\Lambda, \mu ; \mathrm{P}, \mathrm{I})$ are isometrically isomorphic Banach *-algebras.

EXAMPLE 13.2. Consider the locally compact group extension

$$
0 \rightarrow \mathbf{Z} \rightarrow \mathbf{R} \rightarrow \mathrm{G} \rightarrow 0
$$

where $G$ is the circle group identified with $[0,1)$. Then $A=L^{1}(\mathbb{Z}), N=\mathbb{Z}$, $H=\mathbb{R}$ and $\tau_{x}(a)=a, x \in H, a \varepsilon A$, since $H$ is abelian and hence, $\delta$ is trivial. The quadruple $(\mathrm{A}, \mathrm{N}, \mathrm{H}, \tau)$ forms the ingredients for a homogeneous Banach *-algebraic bundle $(A, \pi ; \cdot, *)$ as above. Observe also that $G$ is locally compact and there exists a measurable cross-section $\sigma: G \rightarrow H$ given by the canonical injection of $[0,1)$ into $\mathbb{R}$. It then follows from the results of Section 11 that there also exists an isomorphic Leptin bundle $(\mathrm{G}, \mathrm{A} ; \mathrm{T}, \mathrm{W})$ such that the corresponding algebras $\mathrm{L}^{1}(\mathrm{G}, \mathrm{A} ; \mathrm{T}, \mathrm{W})$ and $\mathrm{L}^{1}(\mathrm{~A}, \mu ; \mathrm{P}, \mathrm{I})$ are (isometrically) 1somorphic. In turn, these are isomorphic to $\mathrm{L}^{1}(\mathrm{H})$. Finally, we leave it to the reader to verify that the Leptin bundle for this example is precisely the one described in 12.9.

\section{APPENDIX}

In this Appendix, we state those properties of multi-linear mappings which will be used in Section 3. All proofs are left to the reader.

Let $A_{1}, \ldots, A_{n}, B$ be Banach spaces. Suppose

$$
\Phi: A_{1} \times \ldots \times A_{n} \rightarrow B
$$

is an $n$-linear mapping. Define

$$
\|\Phi\|=\sup \left\{\frac{\left\|\Phi\left(a_{1}, \ldots, a_{n}\right)\right\|}{\left\|a_{1}\right\| \ldots\left\|a_{n}\right\|}: a_{i} \varepsilon A_{i}, a_{i} \neq 0,1 \leq i \leq n\right\} .
$$

PROPOSITION Al. Suppose $\Phi$ is bounded, i.e. $\|\Phi\|<\infty$. Then:

(i) $\left\|\Phi\left(a_{1}, \ldots, a_{n}\right)\right\| \leq\|\Phi\|\left\|a_{1}\right\| \ldots\left\|a_{n}\right\|$.

(ii) $\|\Phi\|=\sup \left\{\left\|\Phi\left(a_{1}, \ldots, a_{n}\right)\right\|: a_{i} \varepsilon A_{i},\left\|a_{i}\right\|=1,1 \leq i \leq n\right\}$.

(iii) $\| \Phi\left(a_{1}, \ldots, a_{n}\right)-\Phi\left(b_{1}, \ldots, b_{n}\|\leq\| \Phi \|\left[\left\|a_{1}-b_{1}\right\| \cdot\left\|a_{2}\right\| \ldots\left\|a_{n}\right\|+\right.\right.$

$$
\left.+\left\|b_{1}\right\| \cdot\left\|a_{2}-b_{2}\right\| \cdot\left\|a_{3}\right\| \ldots\left\|a_{n}\right\|+\ldots+\left\|b_{1}\right\| \ldots\left\|_{b_{n-1}}\right\| \cdot\left\|a_{n}-b_{n}\right\|\right],
$$


for $a_{1}, b_{1} \varepsilon_{A_{1}} ; \ldots ; a_{n}, b_{n} \varepsilon_{n}$.

LEMMA A2. Let $\mathrm{m}$ be a positive integer,

$$
\left\{a_{k}{ }^{i_{k}}: 1 \leq i_{k} \leq m\right\} \quad \leq A_{k}
$$

and

$$
\left\{\alpha_{k}{ }_{k}{ }_{k} 1 \leq i_{k} \leq m\right\} \subseteq \mathbb{c}, k=1, \ldots, n
$$

Then

$$
\Phi\left(\sum_{\substack{i_{1} \\ \alpha_{1}}{ }^{i_{1}}{ }^{i_{1}} 1}, \ldots, \sum_{i_{n}} \alpha_{n}^{\left.i_{n} a_{n}{ }^{i_{n}}\right)}=\sum_{i_{1}} \alpha_{1}^{i_{1}} \ldots \sum_{i_{n}} \alpha_{n}^{i_{n}} \Phi\left(a_{1}{ }^{i_{1}}, \ldots, a_{n}{ }^{i}\right)\right.
$$

THEOREM A3. The space $\operatorname{Hom}^{\mathrm{n}}\left(\mathrm{A}_{1}, \ldots, \mathrm{A}_{\mathrm{n}} ; \mathrm{B}\right)$ of $\mathrm{n}$-linear mappings $\Phi$ as above which are bounded is a Banach space relative to the norm $\|\Phi\|$.

ACKNOWLEDGEMENT. The author was partially supported by an Oakland University Research Fellowship.

\section{REFERENCES}

1. J.M.G. FELL, An extension of Mackey's method to Banach *-algebraic bundles, Memoirs Amer. Math. Soc., No. 90(1969).

J.M.G. FELL, Induced representations and Banach *-algebraic bundles (with appendix by A. Douady and L. Dal Soglio-Hérault), Lect. Notes Math. 528(1977), Springer-Verlag, New York.

3. H. LEPTIN, Verallgemeinerte $\mathrm{L}^{1}$-algebren, Math. Ann. 159(1965), 51-76.

4. R.C. BUSBY and H.A. SMITH, Representations of twisted group algebras, Trans. Amer. Math. Soc., 149(1970), 503-537.

5. M. LEINERT, Fell bündel und verallgemeinerte $\mathrm{L}^{1}$-algebren, J. Func. Anal. 22 $(1976), 323-345$.

6. M.A. RIEFFEL, Review of 2 above, Bu11. Amer. Math. Soc. (New Series) 1(1979), 232-237.

7. R. GODFMENT, Sur la théorie des représentations unitaires, Ann. Math. 53(1951), 68-124.

8. I.E. SCHOCHETMAN, Kernels and integral operators for continuous sums of Banach spaces, Memoirs Amer. Math. Soc., No. 202, 1978.

9. J.M.G. FELL, The structure of algebras of operator fields, Acta Math. 106(1961), 233-280.

10. N. BOURBAKI, Intégration, Chap. 1-4, deuxième éd., Hermann, Paris, 1965.

11. D. HUSEMOLLER, Fibre bundles, 1966, McGraw-Hi11, New York. 
12. L.H. LOOMIS, An indroduction to abstract harmonic analysis, 1953, D. Van Nostrand, New York.

13. I.E. SCHOCHETMAN, Integral operators in the theory of induced Banach representations, Memoirs Amer. Math. Soc., No. 207, 1978.

14. N. BOURBAKI, Intégration, Chap. 5, deuxième éd., Hermann, Paris, 1967.

15. N. BOURBAKI, Intégration, Chap. 7-8, première éd., Hermann, Paris, 1963.

16. R.A. FONTENOT and I.E. SCHOCHETMAN, Induced representations of groups on Banach spaces, Rocky Mtn. J. Math. 7(1977), 53-82. 


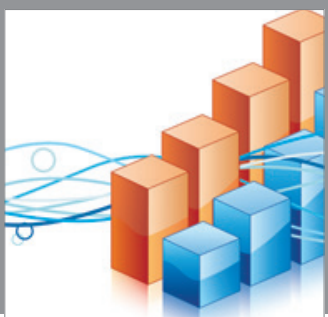

Advances in

Operations Research

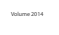

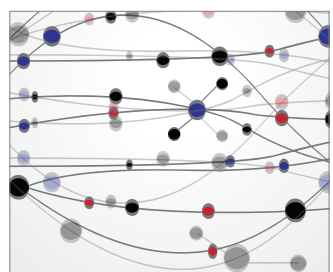

\section{The Scientific} World Journal
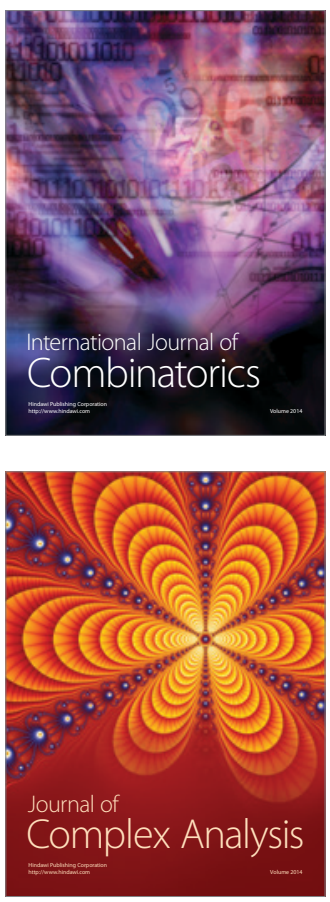

International Journal of

Mathematics and

Mathematical

Sciences
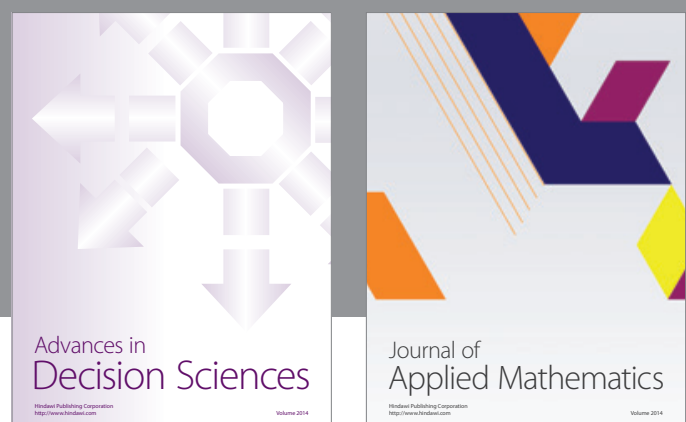

Journal of

Applied Mathematics
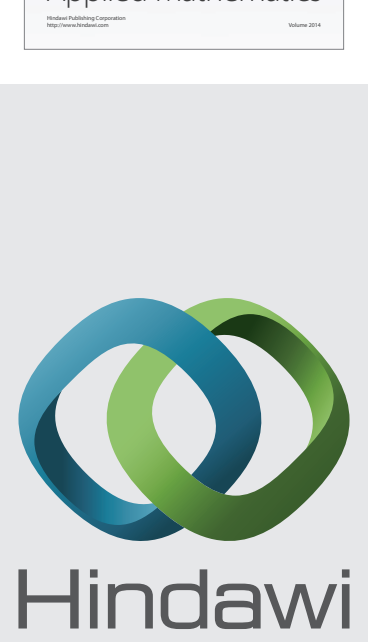

Submit your manuscripts at http://www.hindawi.com
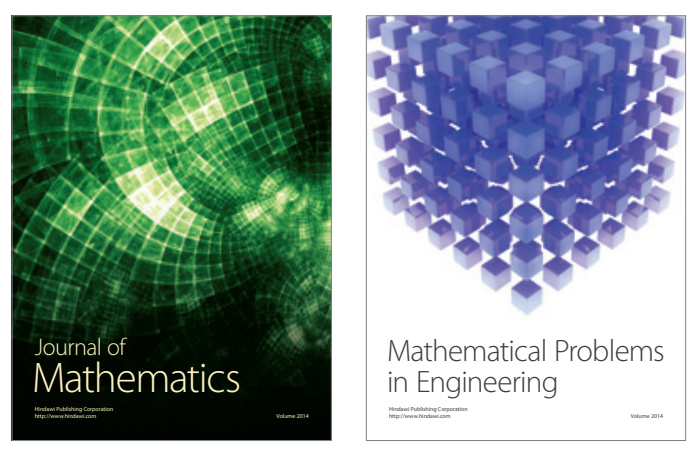

Mathematical Problems in Engineering
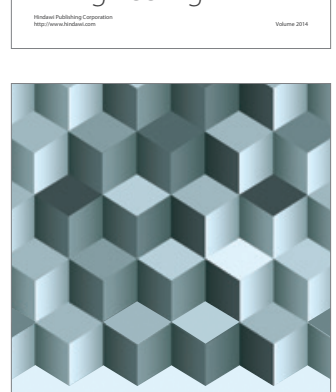

Journal of

Function Spaces
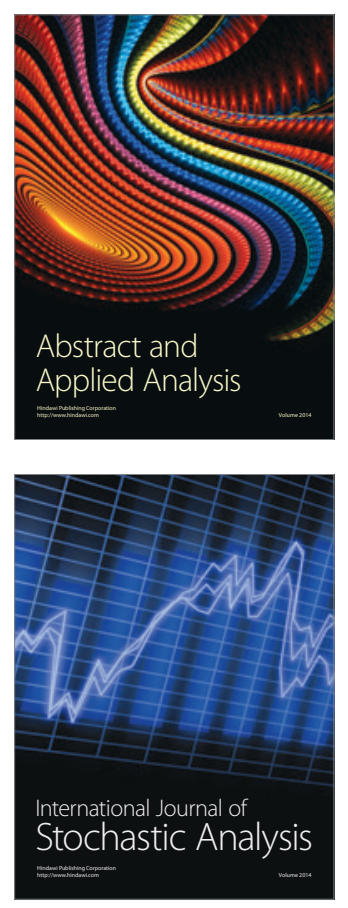

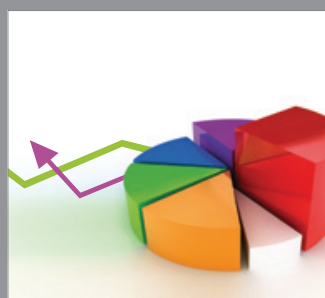

ournal of

Probability and Statistics

Promensencen
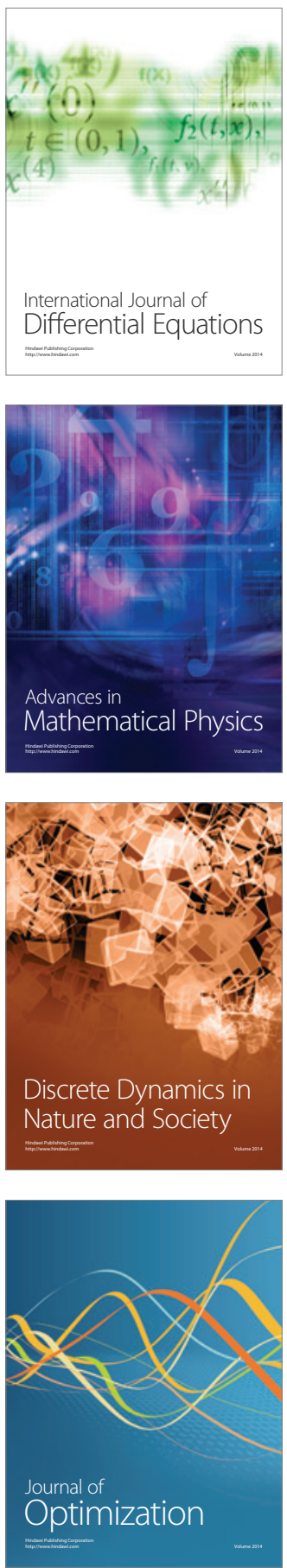